\title{
A computational inverse method for identification of non-Gaussian random fields using the Bayesian approach in very high dimension ${ }^{\text {th }}$
}

\author{
C. Soize* \\ Université Paris-Est, Laboratoire Modélisation et Simulation Multi-Echelle, MSME UMR 8208 \\ CNRS, 5 bd Descartes, 77454 Marne-la-Vallée Cedex 2, France
}

\begin{abstract}
This paper is devoted to the identification of Bayesian posteriors for the random coefficients of the high-dimension polynomial chaos expansions of non-Gaussian tensor-valued random fields using partial and limited experimental data. The experimental data sets correspond to an observation vector which is the response of a stochastic boundary value problem depending on the tensor-valued random field which has to be identified. So an inverse stochastic problem must be solved to perform the identification of the random field. A complete methodology is proposed to solve this very challenging problem in high dimension, which consists in using the first four steps introduced in a previous paper, followed by the identification of the posterior model. The steps of the methodology are the following: (1) introduction of a family of Prior Algebraic Stochastic Model (PASM), (2) identification of an optimal PASM in the constructed family using the partial experimental data, (3) construction of a statistical reduced-order optimal PASM, (4) construction, in high dimension, of the polynomial chaos expansion with deterministic vector-valued coefficients of the reduced-order optimal PASM, (5) substitution of these deterministic vector-valued coefficients by random vector-valued coefficients in order to extend the capability of the polynomial chaos expansion to represent the experimental data and for which the joint probability distribution must be identified, (6) construction of the prior probability model of these
\end{abstract}

\footnotetext{
Computer Methods in Applied Mechanics and Engineering, 200(45-46), 3083-3099 (2011), doi: 10.1016/j.cma.2011.07.005.

${ }^{*}$ Corresponding author

Email address: christian.soize@univ-paris-est.fr(C. Soize)
} 
random vector-valued coefficients and finally, (7) identification of the posterior probability model of these random vector-valued coefficients using partial and limited experimental data, through the stochastic boundary value problem. Two methods are proposed to carry out the identification of the posterior model. The first one is based on the use of the classical Bayesian method. The second one is a new approach derived from the Bayesian method, which is more efficient in high dimension. An application is presented for which several millions of random coefficients are identified.

Key words: Inverse problem, non-Gaussian, random field, Bayes, Bayesian method, identification.

\section{Introduction}

The problem related to the identification of vector-valued parameter of a system modeled by a boundary value problem (BVP) (for instance, the coefficients of a partial differential equation) using experimental data related to the vector-valued observation of this system, is a difficult problem which has been studied a lot. In general and in the deterministic context, there is not a unique solution because the function which maps the vector-valued parameter to the vector-valued observation is not an injection, and consequently, cannot be inverted. It is an ill-posed problem. However, such a problem can be reformulated in terms of an optimization problem consisting in calculating an optimal value of the vector-valued parameter which minimizes a certain distance between the observed experimental data and the vector-valued observation which is computed with the BVP and which depends on the vector-valued parameter (see for instance [54]). In many cases, the analysis of such an inverse problem can have a unique solution in the framework of statistics, that is to say when the vector-valued parameters is modeled by a random quantity, with or without external noise on the observed output. In such a case, the random vector-valued observation is completely defined by its probability distribution which is the unique transformation of the probability distribution of the random vector-valued parameter. This transformation is defined by the function which maps the vector-valued parameter to the vector-valued observation. With such a formulation which becomes a well-posed problem, there is a unique solution in the probability theory framework (see for instance [24] an overview concerning the stochastic inverse problems).

The identification of non-Gaussian random fields solving stochastic inverse prob- 
lems has already been addressed in many scientific and technical areas (see for instance $[13,16,27,53,23,24,28])$.

The present paper is a companion work of the recent paper published in [48] and which was devoted to the identification of the random Vector-Valued Coefficients (VVC) of the high-dimension Polynomial Chaos Expansion (PCE) of a non-Gaussian tensor-valued random field using partial and limited experimental data. These experimental data are related to an observation vector which is the response of a stochastic boundary value problem depending on the tensor-valued random field which has to be identified. A complete new methodology has been proposed to solve this challenging problem in high dimension, in particular new algorithms have been proposed to identify the construction, in high dimension, of the PCE with deterministic VVC of the reduced-order optimal prior algebraic stochastic model of the tensor-valued random field. In order to extend the capability of the PCE to represent the experimental data, the deterministic VVC have been replaced by random VVC for which the joint probability distribution has to be identified. In [48], we have proposed a first approach to construct the prior probability model of these random VVC and then, to identify the posterior probability model of these random VVC using partial and limited experimental data. Nevertheless, an alternative approach seemed to have to be investigated, based on the Bayesian method, as it was indicated in the conclusion of this work. In this paper, we thus present the identification of the Bayesian posteriors of the random VVC of the high-dimension PCE of a non-Gaussian tensor-valued random field using partial and limited experimental data. As we will see, two methods will be developed to perform the identification of the posterior model in high dimension. The first one will be based on the use of the classical Bayesian method. The second one is a new approach derived from the Bayesian method, which is more efficient in high dimension. An application will be presented for which several millions of random coefficients must be identified.

Let us recall that the methodology used to construct the PCE of a random field has been introduced in [17]. The methodologies relative to the PCE of stochastic processes and random fields, and application to stochastic boundary value problems, have generated many works in the last decade (see for instance $[12,18,21,22,25$, $29,30,34,35,36,38,39,41,45,47,55,56])$. The stochastic inverse methods and the Bayesian inference approach to inverse problems have received a particular attention (see for instance [8, 19, 31, 32, 33, 57, 58, 59]). The problem relative to the identification, with experimental data, of the deterministic VVC of the PCE 
of a non-Gaussian real-valued random field using the maximum likelihood has been introduced in [14, 15] and more recently, has been revisited in [11]. In [10], the authors propose to construct the probability model of the random VVC of the PCE by using the asymptotic sampling Gaussian distribution constructed with the Fisher information matrix. Such an approach has been used for model validation $[20,37]$. Recently, in [1], as a continuation of [46], the identification of Bayesian posteriors for the random VVC of PCE has been proposed. Nevertheless, this interesting approach is not perfectly adapted to high-dimension problems (case for which several millions of random variables have to be identified). This is the reason why we propose to explore in this paper, as a continuation of [48], another way for the high-dimension case in the field of the Bayesian inverse method.

\section{Definition of the problem to be solved}

In this section, we recall the challenging problem introduced and solved in [48], for which the last step of the methodology which is devoted to the identification of the posterior probability model, is revisited in this paper. We propose to use the Bayesian method to identify the posterior probability model. We then have to identify the Bayesian posteriors of high-dimension PCE with random VVC for non-Gaussian tensor-valued random fields using partial and limited experimental data.

(1) Stochastic boundary value problem. We consider a boundary value problem for a vector-valued field $\left\{\mathbf{u}(\mathbf{x})=\left(u_{1}(\mathbf{x}), u_{2}(\mathbf{x}), u_{3}(\mathbf{x})\right), \mathbf{x} \in \Omega\right\}$ defined on an open bounded domain $\Omega$ of $\mathrm{R}^{3}$, with generic point $\mathbf{x}=\left(x_{1}, x_{2}, x_{3}\right)$. This boundary value problem depends on a non-Gaussian fourth-order tensor-valued random field $\{\mathrm{C}(\mathbf{x}), \mathbf{x} \in \Omega\}$ in which $\mathrm{C}(\mathbf{x})=\left\{\mathrm{C}_{i j k \ell}(\mathbf{x})\right\}_{i j k \ell}$, which is unknown and which has to be identified solving an inverse stochastic problem. The boundary $\partial \Omega$ of domain $\Omega$ is written as $\Gamma_{0} \cup \Gamma_{\text {obs }} \cup \Gamma$. Field $\mathbf{u}$ is only experimentally observed on $\Gamma_{\text {obs }}$, which means that the system is partially observed with respect to the available experimental data.

(2) Stochastic finite element approximation of the stochastic boundary value problem. The stochastic boundary value problem (introduced in (1) above) is discretized by the finite element method. Let $\mathcal{I}=\left\{\mathbf{x}^{1}, \ldots, \mathbf{x}^{N_{p}}\right\} \subset \Omega$ be the finite subset of $\Omega$ made up of all the integrations points of the finite elements used in the mesh of $\Omega$. For all $\mathbf{x}$ fixed in $\mathcal{I} \subset \Omega$, the fourth-order tensor-valued random 
variable $\mathrm{C}(\mathbf{x})$ is represented by a real random matrix $[\mathrm{A}(\mathbf{x})]$ such that $[\mathrm{A}(\mathbf{x})]_{I J}=$ $\mathrm{C}_{i j k h}(\mathbf{x})$ with a given adapted correspondence $I=(i, j)$ and $J=(k, h)$. It should be noted that mathematical properties on the matrix-valued random field $\{[\mathrm{A}(\mathbf{x})], \mathbf{x} \in \Omega\}$ are necessary in order to preserve the mathematical properties of the boundary value problem. Let $\mathbf{U}=\left(\mathbf{U}^{\text {obs }}, \mathbf{U}^{\text {nobs }}\right)$ be the random vector with values in $\mathrm{R}^{m}=\mathrm{R}^{m_{\mathrm{obs}}} \times \mathrm{R}^{m_{\text {nobs }}}$ with $m=m_{\mathrm{obs}}+m_{\text {nobs }}$, constituted of some degrees of freedom of the finite element approximation of field $\mathbf{u}$. The $\mathrm{R}^{m_{\text {obs }}}$ valued random vector $\mathbf{U}^{\text {obs }}=\left(U_{1}^{\text {obs }}, \ldots, U_{m_{\text {obs }}}^{\text {obs }}\right)$ is made up of the $m_{\text {obs }}$ observed degrees of freedom for which there are available experimental data (corresponding to the finite element approximation of the trace on $\Gamma_{\text {obs }}$ of random field $\mathbf{u}$ ). Vector $\mathbf{U}^{\text {obs }}$ will be called the observation vector. The $\mathrm{R}^{m_{\text {nobs-valued }} \text { random vec- }}$ tor $\mathbf{U}^{\text {nobs }}=\left(U_{1}^{\text {nobs }}, \ldots, U_{m_{\text {nobs }}}^{\text {nob }}\right)$ is made up of the $m_{\text {nobs }}$ degrees of freedom (of the finite element model) for which no experimental data are available and are introduced for performing the quality assessment of the identification which will be done. The random vector $\mathbf{U}$ appears as the unique deterministic nonlinear transformation of the finite family of $N_{p}$ dependent random matrices $\{[\mathrm{A}(\mathbf{x})], \mathbf{x} \in \mathcal{I}\}$. This set of random matrices can then be represented by a $R^{m_{V}}$-valued random vector $\mathrm{V}=\left(V_{1}, \ldots, V_{m_{\vee}}\right)$. Consequently, the $\mathrm{R}^{m}$-valued random vector $\mathbf{U}$ can be written as

$$
\mathbf{U}=\mathbf{h}(\mathrm{V}) \quad, \quad \mathbf{U}^{\mathrm{obs}}=\mathbf{h}^{\mathrm{obs}}(\mathrm{V}) \quad, \quad \mathbf{U}^{\mathrm{nobs}}=\mathbf{h}^{\mathrm{nobs}}(\mathrm{V})
$$

in which $v \mapsto \mathbf{h}(v)=\left(\mathbf{h}^{\mathrm{obs}}(v), \mathbf{h}^{\text {nobs }}(v)\right)$ is a deterministic nonlinear transformation from $\mathrm{R}^{m_{\vee}}$ into $\mathrm{R}^{m}=\mathrm{R}^{m_{\mathrm{obs}}} \times \mathrm{R}^{m_{\text {nobs }}}$ which can be constructed solving the discretized boundary value problem.

(3) Experimental data sets. It is assumed that $\nu_{\text {exp }}$ experimental data sets are available for the observation vector $\mathbf{U}^{\text {obs }}$. Each experimental data set corresponds to partial experimental data (only the trace of the displacement field on $\Gamma_{\text {obs }}$ is observed) with a limited length ( $\nu_{\exp }$ is relatively small). These $\nu_{\exp }$ experimental data sets correspond to measurements of $\nu_{\text {exp }}$ experimental configurations associated with the same boundary value problem. For configuration $\ell$, with $\ell=1, \ldots, \nu_{\text {exp }}$, the observation vector (corresponding to $\mathbf{U}^{\text {obs }}$ for the computational model) is denoted by $\mathbf{u}^{\exp , \ell}$ and belongs to $\mathrm{R}^{m}$. Therefore, the available data are made up of the $\nu_{\text {exp }}$ vectors $\mathbf{u}^{\exp , 1}, \ldots, \mathbf{u}^{\exp , \nu_{\exp }}$ in $\mathrm{R}^{m}$. Below, it is assumed that $\mathbf{u}^{\exp , 1}, \ldots, \mathbf{u}^{\exp , \nu_{\exp }}$ can be viewed as $\nu_{\text {exp }}$ independent realizations of a random vector $\mathbf{U}^{\text {exp }}$ defined on a probability space $\left(\Theta^{\exp }, \mathcal{T}^{\exp }, \mathcal{P}^{\exp }\right)$ and corresponding to random observation vector $\mathbf{U}^{\text {obs }}$ (but noting that random vectors $\mathbf{U}^{\text {exp }}$ and $\mathbf{U}^{\text {obs }}$ are not defined on the 
same probability space).

(4) Stochastic inverse problem to be solved. The problem to be solved concerns the identification of the unknown non-Gaussian random vector $\mathrm{V}$ representing the fourth-order tensor-valued random field $\{\mathrm{C}(\mathbf{x}), \mathbf{x} \in \Omega\}$, using partial and limited experimental data $\mathbf{u}^{\exp , 1}, \ldots, \mathbf{u}^{\exp , \nu_{\exp }}$ relative to the random observation vector $\mathbf{U}^{\text {obs }}$ such that $\mathbf{U}^{\text {obs }}=\mathbf{h}^{\text {obs }}(\mathrm{V})$ in which $\mathbf{h}^{\text {obs }}$ is a given deterministic nonlinear mapping. The components of the random vector $\mathbf{U}^{\text {nobs }}$, such that $\mathbf{U}^{\text {nobs }}=\mathbf{h}^{\text {nobs }}(\mathrm{V})$ in which $\mathbf{h}^{\text {nobs }}$ is a given deterministic nonlinear mapping, are used for performing the quality assessment of the identification .

\section{Summarizing the methodology previously introduced to identify a high- dimension PCE using partial and limited experimental data}

The identification of Bayesian posteriors of high-dimension PCE with random $\mathrm{VVC}$, using partial and limited experimental data, requires a first identification of the deterministic VVC of the high-dimension PCE for the non-Gaussian tensorvalued random field, using partial and limited experimental data. Such a first identification, performed in four steps, is described in details in [48]. In this section, we briefly summarize the methodology for readability of the paper.

Step 1. Introduction of a family of Prior Algebraic Stochastic Models (PASM) for random vector $\mathrm{V}$. The available partial and limited experimental data are not sufficient to perform a direct statistical estimation of the covariance matrix $\left[C_{\mathrm{V}}\right]$, that would be necessary to construct a reduced-order statistical model deduced from the Karhunen-Loeve expansion of the random field $\{[\mathrm{A}(\mathbf{x})], \mathbf{x} \in \Omega\}$ (that is to say deduced from a principal component analysis of random vector $\mathrm{V}$ ). In addition, such a reduced-order statistical model must have the capability to represent the required mathematical properties for the random family $\left\{\left[\mathrm{A}\left(\mathbf{x}^{1}\right)\right], \ldots,\left[\mathrm{A}\left(\mathbf{x}^{N_{p}}\right)\right]\right\}$ (for instance, each random matrix $\left[\mathrm{A}\left(\mathbf{x}^{k}\right)\right]$ should be positive definite almost surely). To circumvent these two major difficulties, it was proposed to introduce a family $\left\{\left[\AA^{\mathrm{PASM}}(\mathbf{x} ; \mathbf{w})\right], \mathbf{x} \in \Omega\right\}_{\mathbf{w}}$ of Prior Algebraic Stochastic Models (PASM) to represent the matrix-valued random field $\{[\mathrm{A}(\mathbf{x})], \mathbf{x} \in \Omega\}$. We can then deduce a family $\left\{\mathrm{V}^{\mathrm{PASM}}(\mathbf{w})\right\}_{\mathbf{w}}$ of PASM for random vector $\mathrm{V}$. This family is defined on a probability space $(\Theta, \mathcal{T}, \mathcal{P})$ and depends on the vector-valued parameter $\mathbf{w}$ belonging to an admissible set $\mathcal{C}_{\text {ad }}$. The knowledge of such a family means that the family $\left\{P_{\mathrm{V}}^{\mathrm{PASM}}(d v ; \mathbf{w}), \mathbf{w} \in \mathcal{C}_{\mathrm{ad}}\right\}$ of probability distributions on $\mathrm{R}^{m_{\vee}}$ of the family of random vectors $\left\{\mathrm{V}^{\mathrm{PASM}}(\mathbf{w}), \mathbf{w} \in \mathcal{C}_{\mathrm{ad}}\right\}$ is known. In addition, it is assumed that 
a generator of $\nu_{\mathrm{KL}}$ independent realizations $\mathrm{V}^{\mathrm{PASM}}\left(\theta_{1} ; \mathbf{w}\right), \ldots, \mathrm{V}^{\mathrm{PASM}}\left(\theta_{\nu_{\mathrm{KL}}} ; \mathbf{w}\right)$ for $\theta_{1}, \ldots, \theta_{\nu_{\mathrm{KL}}}$ belonging to $\Theta$ is available. In practice, vector-valued parameter $\mathbf{w}$ will be chosen as a vector with a very low dimension and its components will be, for instance, the mean value $[\underline{\mathrm{A}}]$, the spatial correlation lengths and the dispersion parameters controlling the statistical fluctuations of matrix-valued random field $\{[\mathrm{A}(\mathbf{x})], \mathbf{x} \in \Omega\}$.

Step 2. Identification of an optimal PASM in the constructed family using the experimental data sets. This step consists in using the experimental data $\left\{\mathbf{u}^{\exp , 1}, \ldots\right.$, $\left.\mathbf{u}^{\exp , \nu \exp }\right\}$ to identify the optimal value $\mathbf{w}^{\text {opt }}$ of parameter $\mathbf{w}$. Using the computational model (see Eq. (1)) and the family $\mathrm{V}^{\mathrm{PASM}}(\mathbf{w})$ of PASM for V, we can construct the family $\left\{\mathbf{U}^{\text {obs,PASM }}(\mathbf{w}), \mathbf{w} \in \mathcal{C}_{\text {ad }}\right\}$ of random observation vectors such that $\mathbf{U}^{\text {obs,PASM }}(\mathbf{w})=\mathbf{h}^{\text {obs }}\left(\mathrm{V}^{\mathrm{PASM}}(\mathbf{w})\right)$ for $\mathbf{w} \in \mathcal{C}_{\mathrm{ad}}$. The optimal PASM is then obtained in finding the optimal value $\mathbf{w}^{\text {opt }}$ of $\mathbf{w}$ which minimizes an adapted "distance" $J(\mathbf{w})$ (cost function) between the family $\left\{\mathbf{U}^{\text {obs,PASM }}(\mathbf{w}), \mathbf{w} \in \mathcal{C}_{\text {ad }}\right\}$ of random observation vectors and the family of experimental data $\left\{\mathbf{u}^{\exp , 1}, \ldots, \mathbf{u}^{\exp , \nu_{\exp }}\right\}$. We then obtain the optimal PASM denoted by $\mathrm{V}^{\mathrm{PASM}}=\mathrm{V}^{\mathrm{PASM}}\left(\mathbf{w}^{\text {opt }}\right)$. Several methods can be used to define the cost function $J(\mathbf{w})$, such as the moment method, the least-square method, the maximum likelihood method, etc (see $[40,50,54]$ ). By construction of the PASM, the dimension of vector $\mathbf{w}$ is much smaller than $\nu_{\text {exp }} \times m_{\text {obs. }}$. The least-square method or the maximum likelihood method can be used to calculate the optimal value $\mathbf{w}^{\text {opt }}$ (see the details in [48]).

Step 3. Construction of the statistical reduced-order optimal PASM. For $\ell=$ $1, \ldots, \nu_{\mathrm{KL}}$, let $\mathrm{V}^{\mathrm{PASM}}\left(\theta_{\ell}\right)$ be $\nu_{\mathrm{KL}}$ independent realizations of the optimal PASM $\mathrm{V}^{\mathrm{PASM}}$. The mean value $\underline{\mathrm{V}}=E\left\{\mathrm{~V}^{\mathrm{PASM}}\right\}$ of $\mathrm{V}^{\mathrm{PASM}}$ ( $E$ is the mathematical expectation) and its positive-definite symmetric $\left(m_{\mathrm{V}} \times m_{\mathrm{V}}\right)$ real covariance matrix $\left[C_{\mathrm{V}} \mathrm{PASM}\right]=E\left\{\left(\mathrm{~V}^{\mathrm{PASM}}-\underline{\mathrm{V}}\right)\left(\mathrm{V}^{\mathrm{PASM}}-\underline{\mathrm{V}}\right)^{T}\right\}$ are estimated using independent realizations $\left\{\mathrm{V}^{\mathrm{PASM}}\left(\theta_{\ell}\right), \ell=1, \ldots \nu_{\mathrm{KL}}\right\}$. The dominant eigenspace of the eigenvalue problem $\left[C_{\mathrm{V}_{\text {PASM }}}\right] \mathrm{W}^{j}=\lambda_{j} \mathrm{~W}^{j}$ is then constructed. Let $[\mathrm{W}]=\left[\mathrm{W}^{1} \ldots \mathrm{W}^{n}\right]$ be the $\left(m_{\mathrm{V}} \times n\right)$ real matrix of the $n$ eigenvectors associated with the $n$ largest eigenvalues $\lambda_{1} \geq \lambda_{2} \geq \ldots \geq \lambda_{n}>0$ such that $[\mathrm{W}]^{T}[\mathrm{~W}]=\left[I_{n}\right]$, in which $\left[I_{n}\right]$ is the $(n \times n)$ identity matrix. The statistical reduced-order optimal PASM is then written as

$$
\mathrm{V}^{\mathrm{PASM}} \simeq \underline{\mathrm{V}}+\sum_{j=1}^{n} \sqrt{\lambda_{j}} \eta_{j}^{\mathrm{PASM}} \mathrm{W}^{j},
$$

in which $\boldsymbol{\eta}^{\mathrm{PASM}}=\left(\eta_{1}^{\mathrm{PASM}}, \ldots, \eta_{n}^{\mathrm{PASM}}\right)$ is a second-order centered random variable 
with values in $\mathrm{R}^{n}$ such that

$$
E\left\{\boldsymbol{\eta}^{\mathrm{PASM}}\right\}=\mathbf{0} \quad, \quad E\left\{\boldsymbol{\eta}^{\mathrm{PASM}}\left(\boldsymbol{\eta}^{\mathrm{PASM}}\right)^{T}\right\}=\left[I_{n}\right] .
$$

The mean-square convergence of the right-hand side in Eq. (2)) with respect to the reduced-order $n$ is studied in constructing the error function

$$
n \mapsto \operatorname{err}(n)=1-\frac{\sum_{j=1}^{n} \lambda_{j}}{\operatorname{tr}\left[C_{\mathrm{V}^{\mathrm{PASM}}}\right]},
$$

which is a monotonic decreasing function from $\left\{1, \ldots, m_{\mathrm{V}}\right\}$ into $[0,1]$ and such that $\operatorname{err}\left(m_{\mathrm{V}}\right)=0$. The $\nu_{\mathrm{KL}}$ independent realizations $\boldsymbol{\eta}^{\mathrm{PASM}}\left(\theta_{1}\right), \ldots, \boldsymbol{\eta}^{\mathrm{PASM}}\left(\theta_{\nu_{\mathrm{KL}}}\right)$ are deduced from the realizations $\mathrm{V}^{\mathrm{PASM}}\left(\theta_{1}\right), \ldots, \mathrm{V}^{\mathrm{PASM}}\left(\theta_{\nu_{\mathrm{KL}}}\right)$ using, for $j=1, \ldots, n$ and for $\ell=1, \ldots \nu_{\mathrm{KL}}$, the equation

$$
\eta_{j}^{\mathrm{PASM}}\left(\theta_{\ell}\right)=\frac{1}{\sqrt{\lambda_{j}}}\left(\mathrm{~V}^{\mathrm{PASM}}\left(\theta_{\ell}\right)-\underline{\mathrm{V}}\right)^{T} \mathrm{~W}^{j}
$$

Step 4. Construction of the PCE with deterministic VVC of the reduced-order optimal PASM. This step consists in constructing an approximation $\boldsymbol{\eta}^{\text {chaos }}(N)=$ $\left(\eta_{1}^{\text {chaos }}(N), \ldots, \eta_{n}^{\text {chaos }}(N)\right)$ of $\boldsymbol{\eta}^{\text {PASM }}$ by using a PCE, such that

$$
\boldsymbol{\eta}^{\mathrm{PASM}} \simeq \boldsymbol{\eta}^{\text {chaos }}(N) \quad, \quad \boldsymbol{\eta}^{\text {chaos }}(N)=\sum_{\alpha=1}^{N} \mathbf{y}^{\alpha} \Psi_{\alpha}(\boldsymbol{\Xi})
$$

in which the real valued random variables $\Psi_{1}(\boldsymbol{\Xi}), \ldots, \Psi_{N}(\boldsymbol{\Xi})$ are the renumbered normalized Hermite polynomials of the $\mathrm{R}^{N_{g}}$-valued normalized Gaussian random variable $\boldsymbol{\Xi}=\left(\Xi_{1}, \ldots, \Xi_{N_{g}}\right)$ (therefore, $E\{\boldsymbol{\Xi}\}=\mathbf{0}$ and $E\left\{\boldsymbol{\Xi} \boldsymbol{\Xi}^{T}\right\}=\left[I_{N_{g}}\right]$ ), defined on probability space $(\Theta, \mathcal{T}, \mathcal{P})$, such that for all $\alpha$ and $\beta$ in $\{1, \ldots, N\}$,

$$
E\left\{\Psi_{\alpha}(\boldsymbol{\Xi})\right\}=0 \quad, \quad E\left\{\Psi_{\alpha}(\boldsymbol{\Xi}) \Psi_{\beta}(\boldsymbol{\Xi})\right\}=\delta_{\alpha \beta} \quad,
$$

where $\delta_{\alpha \beta}$ is the Kronecker symbol. It should be noted that the constant Hermite polynomial with index $\alpha=0$ is not included in Eq. (6). If $N_{d}$ is the integer number representing the maximum degree of the Hermite polynomials, then the number $N$ of chaos in Eq. (6) is

$$
N=h\left(N_{g}, N_{d}\right)=\left(N_{d}+N_{g}\right) ! /\left(N_{d} ! N_{g} !\right)-1 .
$$


In Eq. (6)), the symbol " $\cong "$ means that the mean-square convergence is reached for $N$ sufficiently large and the deterministic VVC which must be identified are the $N$ vectors $\mathbf{y}^{1}, \ldots, \mathbf{y}^{N}$ in $\mathrm{R}^{n}$. Taking into account Eqs. (3) and (7), it can be deduced that vectors $\mathbf{y}^{1}, \ldots, \mathbf{y}^{N}$ must verify the following equation,

$$
\sum_{\alpha=1}^{N} \mathbf{y}^{\alpha} \mathbf{y}^{\alpha T}=\left[I_{n}\right]
$$

In order to control the quality of the convergence of the series in Eq. (6) with respect to $N$ (which is mean-square convergent), we have introduced in [48] an unusual $L^{1}-\log$ error function which allows the errors of the very small values of the probability density function (the tails of the probability density function) to be measured. For a fixed value of $N$, such a measurement of the error is summarized hereinafter. Let $e \mapsto p_{j}^{\text {PASM }}(e)$ be the probability density function of the random variable $\eta_{j}^{\text {PASM }}$. For all $\mathbf{y}^{1}, \ldots, \mathbf{y}^{N}$ fixed in $\mathrm{R}^{n}$ and satisfying Eq. (9)), let $e \mapsto p_{\eta_{j}^{\text {chaos }}(N)}\left(e ; \mathbf{y}^{1}, \ldots, \mathbf{y}^{N}\right)$ be the probability density function of random variable $\eta_{j}^{\text {chaos }}(N)$. The convergence of the sequence of random vectors $\left\{\boldsymbol{\eta}^{\text {chaos }}(N)\right\}_{N}$ towards $\boldsymbol{\eta}^{\mathrm{PASM}}$ is then controlled with the $L^{1}-\log$ error defined by

$$
\operatorname{err}_{j}\left(N_{g}, N_{d}\right)=\int_{\mathrm{BI}_{j}}\left|\log _{10} p_{\eta_{j}^{\mathrm{PASM}}}(e)-\log _{10} p_{\eta_{j}^{\text {chaos }}(N)}\left(e ; \mathbf{y}^{1}, \ldots, \mathbf{y}^{N}\right)\right| d e
$$

in which $\mathrm{BI}_{j}$ is a bounded interval of the real line which is adapted to the problem (see the details in [48]). The estimation of $p_{\eta_{j}^{\mathrm{PASM}}}(e)$ is carried out using the kernel density estimation method [6] with the independent realizations $\eta_{j}^{\mathrm{PASM}}\left(\theta_{1}\right), \ldots$, $\eta_{j}^{\mathrm{PASM}}\left(\theta_{\nu_{\mathrm{KL}}}\right)$ calculated in Step 3. Similarly, for a given value of $\mathbf{y}^{1}, \ldots, \mathbf{y}^{N}$, the estimation of $p_{\eta_{j}^{\text {chaos }}(N)}\left(e ; \mathbf{y}^{1}, \ldots, \mathbf{y}^{N}\right)$ is carried out using Eq. (6) and $\nu$ independent realizations $\boldsymbol{\Xi}\left(\theta_{1}\right), \ldots, \boldsymbol{\Xi}\left(\theta_{\nu}\right)$ of the normalized Gaussian vector $\boldsymbol{\Xi}$ defined on probability space $(\Theta, \mathcal{T}, \mathcal{P})$ with $\theta_{1}, \ldots, \theta_{\nu}$ in $\Theta$. For the random vector $\boldsymbol{\eta}^{\text {chaos }}(N)$, the $L^{1}-\log$ error function is denoted as $\operatorname{err}\left(N_{g}, N_{d}\right)$ and is defined by

$$
\operatorname{err}\left(N_{g}, N_{d}\right)=\frac{1}{n} \sum_{j=1}^{n} \operatorname{err}_{j}\left(N_{g}, N_{d}\right)
$$

It should be noted that Eqs. (10) and (11) are not used to identify $\mathbf{y}^{1}, \ldots, \mathbf{y}^{N}$, but only to evaluate, for each fixed value of $N$ and for given $\mathbf{y}^{1}, \ldots, \mathbf{y}^{N}$, the quality of the approximation $\boldsymbol{\eta}^{\text {PASM }} \simeq \boldsymbol{\eta}^{\text {chaos }}(N)$. For each fixed value of $N$, the identification of $\mathbf{y}^{1}, \ldots, \mathbf{y}^{N}$ is performed using the maximum likelihood method 
$[40,50,54]$ as done in $[14,15,10,48]$. Taking into account that the dependent random variables $\eta_{1}^{\mathrm{PASM}}, \ldots, \eta_{n}^{\mathrm{PASM}}$ are not correlated, the following approximation $\mathcal{L}\left(\mathbf{y}^{1}, \ldots, \mathbf{y}^{N}\right)$ of the log-likelihood function is introduced

$$
\mathcal{L}\left(\mathbf{y}^{1}, \ldots, \mathbf{y}^{N}\right)=\sum_{j=1}^{n} \sum_{\ell=1}^{\nu_{\mathrm{KL}}} \log _{10} p_{\eta_{j}^{\text {chaos }}(N)}\left(\eta_{j}^{\mathrm{PASM}}\left(\theta_{\ell}\right) ; \mathbf{y}^{1}, \ldots, \mathbf{y}^{N}\right)
$$

The optimal value $\left(\underline{\mathbf{y}}^{1}, \ldots, \underline{\mathbf{y}}^{N}\right)$ of $\left(\mathbf{y}^{1}, \ldots, \mathbf{y}^{N}\right)$ is then given by

$$
\left(\underline{\mathbf{y}}^{1}, \ldots, \underline{\mathbf{y}}^{N}\right)=\arg \max _{\left(\mathbf{y}^{1}, \ldots, \mathbf{y}^{N}\right) \in \mathcal{C}_{\mathrm{ad}}^{N}} \mathcal{L}\left(\mathbf{y}^{1}, \ldots, \mathbf{y}^{N}\right)
$$

in which $\mathcal{C}_{\text {ad }}^{N}$ is such that

$$
\mathcal{C}_{\mathrm{ad}}^{N}=\left\{\left(\mathbf{y}^{1}, \ldots, \mathbf{y}^{N}\right) \in\left(\mathrm{R}^{n}\right)^{N}, \sum_{\alpha=1}^{N} \mathbf{y}^{\alpha} \mathbf{y}^{\alpha T}=\left[I_{n}\right]\right\} .
$$

For the high-dimension case, that is to say for $n \times N$ very large, solving the optimization problem defined by Eqs. (13) and (14) is a very challenging problem which has been solved in the last decade only for small values of $n$ and $N$. Such a challenging problem has been solved in [48] thanks to the use of two novel algorithms:

(i) The first one is required, for $\alpha=1, \ldots, N$ and $\ell=1, \ldots, \nu$, to generate the independent realizations $\Psi_{\alpha}\left(\boldsymbol{\Xi}\left(\theta_{\ell}\right)\right)$ of $\Psi_{\alpha}(\boldsymbol{\Xi})$ with high degree $N_{d}$ of the polynomials $\Psi_{\alpha}$. Introducing the $(\nu \times N)$ real matrix $[\Psi]$ such that $[\Psi]_{\ell \alpha}=\Psi_{\alpha}\left(\Xi\left(\theta_{\ell}\right)\right)$, matrix $[\Psi]$ is computed as explained in [49] to preserve the orthogonality conditions defined by Eq. (7) for any values of $N_{g}$ and $N_{d}$.

(ii) The details of the second one are given in [48] and allows the high-dimension optimization problem defined by Eqs. (13) and (14) to be solved with a reasonable CPU time, the constraint defined by Eq. (9) being automatically and exactly satisfied.

(iii) The random response vector $\mathbf{U}^{\mathrm{PASM}}=\left(\mathbf{U}^{\text {obs,PASM }}, \mathbf{U}^{\text {nobs,PASM }}\right)$ of the computational stochastic model, corresponding to the optimal PASM represented by the $\mathrm{PCE}$, is given by $\mathbf{U}^{\mathrm{PASM}}=\mathbf{h}\left(\mathrm{V}^{\mathrm{PASM}}\right)$ in which $\mathrm{V}^{\mathrm{PASM}} \simeq \underline{\mathrm{V}}+\sum_{j=1}^{n} \sqrt{\lambda_{j}} \eta_{j}^{\mathrm{PASM}} \mathrm{W}^{j}$ with $\boldsymbol{\eta}^{\mathrm{PASM}} \simeq \sum_{\alpha=1}^{N} \underline{\mathbf{y}}^{\alpha} \Psi_{\alpha}(\boldsymbol{\Xi})$. The independent realizations $\left\{\mathbf{U}^{\mathrm{PASM}}\left(\theta_{\ell}\right), \ell=\right.$ 
$1, \ldots, \nu\}$ of $\mathbf{U}^{\mathrm{PASM}}$ can then be calculated. For $1 \leq k \leq m_{\mathrm{obs}}$, let $U_{k}^{\mathrm{PASM}}$ be a component of the random observation vector $\mathbf{U}^{\text {obs,PASM }}$ while, if $m_{\mathrm{obs}}+1 \leq k \leq m$, then $U_{k}^{\text {PASM }}$ represents a component of the random vector $\mathbf{U}^{\text {nobs,PASM }}$. The probability density function $u_{k} \mapsto p_{U_{k}^{\mathrm{PASM}}}\left(u_{k}\right)$ on $\mathrm{R}$ of the random variable $U_{k}^{\mathrm{PASM}}$ is then estimated using the above independent realizations and the kernel density estimation method [6].

\section{Comments about the proposed methodology and identification of Bayesian posteriors for the high-dimension PCE using partial and limited experi- mental data}

For given experimental data sets, the best approach which can be made is the one (1) which takes into account all the available theoretical information related to the tensor-valued random field and (2) which reproduces the set of the available experimental data in a statistical sense. The family of PASM (see Step 1) which depends on the low-dimension vector-valued parameter $\mathbf{w}$ must span the larger possible subset of all the admissible tensor-valued random fields. The construction of such a family must then take into account all the available mathematical properties (for instance an ellipticity condition). Nevertheless, since the optimal PASM (see Step 2) is constructed in identifying a family which has only a few free parameters (components of vector w), this optimal PASM belongs to a subset which is not big enough to perfectly represent the available experimental data. Consequently, it is necessary to construct a posterior model to better represent the experimental data, that is to say, it is necessary to construct a representation which is capable to span a larger subset containing the experimental data. The approach used has then been to construct a PCE with random VVC of the optimal PASM. Such a PCE with random VVC has the capability to represent any tensor-valued random field and then has the capability to fit all the experimental data in a statistical sense. Nevertheless, a direct construction of such PCE with random VVC would not be realistic. This is the reason why, in Step 4, the PCE with deterministic VVC of the optimal PASM is constructed and then, the posterior model is constructed in replacing the deterministic VVC of the PCE by random VVC. Clearly, better will be the optimal PASM, less will be the numerical cost required to fit the probability distributions of the random VVC.

Let us consider an uncertain computational model for which the stochastic modeling of uncertain parameters is performed by introducing $N_{g}$ independent random variables (after having applied a Karhunen-Loeve statistical reduction and a nonlinear transformation of independent random variables for which the probability 
measures are known). Clearly, for the direct problem consisting in analyzing the propagation of uncertainties through the computational model, the length of the germ of the PCE of the random response of this stochastic computational model, must be chosen as $N_{g}$. In this paper, the stochastic inverse problem is considered. The uncertain parameter is a random field for which the stochastic model is unknown and must be identified. Using the Karhunen-Loeve statistical reduction (see Step 3) and then the PCE of the $\mathrm{R}^{n}$-valued random variable $\boldsymbol{\eta}^{\text {PASM }}$ (see Step 4), there are three unknown parameters $n, N_{g}$ and $N_{d}$, which must be selected to get a good convergence of the representation. The value of parameter $n$ is defined in studying the error function $n \mapsto \operatorname{err}(n)$ defined by Eq. (4) which is based on a mean-square convergence. The value of $n$ is then independent from the value of $N_{g}$ and $N_{d}$. Now, the value of $N_{g}$ and $N_{d}$ must be defined in order that the convergence of the PCE of the $\mathrm{R}^{n}$-valued random variable $\boldsymbol{\eta}^{\mathrm{PASM}}$ be reached. Such a convergence is studied using the unusual $L^{1}$-log error function $\left(N_{g}, N_{d}\right) \mapsto \operatorname{err}\left(N_{g}, N_{d}\right)$ defined by Eqs. (10) and (11). It should be noted that this error function is better than the mean-square error function and allows the convergence of the probability function to be controlled over all the range of the large values and the very small values of the probability levels (this means that this error function allows the tail of the probability density functions to be correctly fitted). Consequently, the maximum degree $N_{d}$ of the polynomial chaos must be sufficiently high to get the convergence of the representation when the random field is any non-Gaussian random field (a random field which is unknown and which must be identified without any information about the tails of the system of marginal probability distributions which define the probability law of the non-Gaussian random field). In addition, the introduction of a very large number $N$ of polynomial chaos $\left\{\Psi_{\alpha}(\boldsymbol{\Xi}), \alpha=1, \ldots, N\right\}$ induced by the use of a high value of $N_{d}$ coupled with the use of a significant value of $N_{g}$, is equivalent to the introduction of a very large number of uncorrelated random variables due to the orthogonal property defined by Eq. (7).

For the stochastic inverse problem under consideration, this analysis shows that, there is no reason to set a priori a value for $N_{g}$ or a value for $N_{d}$ which are strongly dependent. The optimal values of $N_{g}$ and $N_{d}$ must be determined using the $L^{1}-\log$ error function and there is no reason to set the value of $N_{g}$ to the value $n$.

As explained above, the family of PASM which is introduced is, in general, not capable to perfectly represent the experimental data (for instance, this can be the case for the mesoscale stochastic modeling of complex anisotropic and heterogeneous microstructures). It should be noted that the family of PASM does not take into account modeling errors but is introduced to model the random medium. 
Consequently, a posterior stochastic model must be introduced in order to improve the optimal PASM which has been constructed and identified in Steps 1 to 3 and for which the representation by a high-dimension PCE has been constructed in Step 4. Such a posterior stochastic model is mainly constructed in order to take into account the modeling errors introduced by the choice of the family of the PASM.

Following the method of reduced PCE with random VVC of vector-valued random variables presented in $[46,48,1]$ and in order to take into account model uncertainties, the optimal values $\left(\underline{\mathbf{y}}^{1}, \ldots, \underline{\mathbf{y}}^{N}\right)$ constructed in Step 4 are replaced by a family of random vectors $\left\{\mathbf{Y}^{1}, \ldots, \mathbf{Y}^{N}\right\}$ defined on a probability space $\left.\left(\Theta^{\prime}, \mathcal{T}^{\prime}, \mathcal{P}^{\prime}\right)\right)$ which is independent of the family of random variables $\left\{\Psi_{1}(\boldsymbol{\Xi}), \ldots, \Psi_{N}(\boldsymbol{\Xi})\right\}$ (which are defined on the probability space $(\Theta, \mathcal{T}, \mathcal{P})$ ).

In [48], we have proposed a first approach to identify such a posterior stochastic model. Presently, we propose an alternative approach based on the use of the Bayesian method for the high-dimension case. The identification of Bayesian posteriors in high-dimension case (that is to say the identification of a posterior probability distribution of a random vector with several millions of dependent components) using a random observation vector for which limited and partial experimental data are available is also a challenging problem.

\section{Prior probability model of the random VVC}

\subsection{Prior model}

Let $\mathrm{V}^{\text {prior }}$ be the prior stochastic model of $\mathrm{V}^{\mathrm{PASM}}$, defined as the $\mathrm{R}^{m_{\mathrm{V}}}$-valued random variable on the probability space $\left(\Theta^{\prime} \times \Theta, \mathcal{T}^{\prime} \otimes \mathcal{T}, \mathcal{P}^{\prime} \otimes \mathcal{P}\right)$, such that

$$
\mathrm{V}^{\text {prior }}=\underline{\mathrm{V}}+\sum_{j=1}^{n} \sqrt{\lambda_{j}} \eta_{j}^{\text {prior }} \mathrm{W}^{j}
$$

The prior stochastic model $\boldsymbol{\eta}^{\text {prior }}=\left(\eta_{1}^{\text {prior }}, \ldots, \eta_{n}^{\text {prior }}\right)$ is a $\mathrm{R}^{n}$-valued random variable defined on $\left(\Theta^{\prime} \times \Theta, \mathcal{T}^{\prime} \otimes \mathcal{T}, \mathcal{P}^{\prime} \otimes \mathcal{P}\right)$, which is written as the following PCE with random VVC (deduced from Eq. (6)),

$$
\boldsymbol{\eta}^{\text {prior }}=\sum_{\alpha=1}^{N} \mathbf{Y}^{\alpha, \text { prior }} \Psi_{\alpha}(\boldsymbol{\Xi})
$$


The family of $\mathrm{R}^{n}$-valued random variables $\left\{\mathbf{Y}^{1, \text { prior }}, \ldots, \mathbf{Y}^{N \text {,prior }}\right\}$ are defined on the probability space $\left(\Theta^{\prime}, \mathcal{T}^{\prime}, \mathcal{P}^{\prime}\right)$. We introduce the random vector $Y^{\text {prior }}$ with values in $\mathrm{R}^{n N}$, defined on $\left(\Theta^{\prime}, \mathcal{T}^{\prime}, \mathcal{P}^{\prime}\right)$, such that

$$
\mathrm{Y}^{\text {prior }}=\left(\mathbf{Y}^{1, \text { prior }}, \ldots, \mathbf{Y}^{N, \text { prior }}\right)
$$

and for which its probability distribution is assumed to be represented by a probability density function $\mathrm{y} \mapsto p_{\mathrm{Y}}^{\text {prior }}(\mathrm{Y})$ on $\mathrm{R}^{n N}$ with respect to the Lebesgue measure $d \mathrm{y}=d \mathrm{y}_{1} \ldots d \mathrm{y}_{n N}$.

\subsection{Independent realizations of the prior model}

For all $\left(\theta^{\prime}, \theta\right)$ in $\Theta^{\prime} \times \Theta$, the realization $\mathrm{V}^{\text {prior }}\left(\theta^{\prime}, \theta\right)$ of $\mathrm{V}^{\text {prior }}$ is written as

$$
\mathrm{V}^{\text {prior }}\left(\theta^{\prime}, \theta\right)=\underline{\mathrm{V}}+\sum_{j=1}^{n} \sqrt{\lambda_{j}} \eta_{j}^{\text {prior }}\left(\theta^{\prime}, \theta\right) \mathrm{W}^{j}
$$

in which the realization $\boldsymbol{\eta}^{\text {prior }}\left(\theta^{\prime}, \theta\right)=\left(\eta_{1}^{\text {prior }}\left(\theta^{\prime}, \theta\right), \ldots, \eta_{n}^{\text {prior }}\left(\theta^{\prime}, \theta\right)\right)$ of $\boldsymbol{\eta}^{\text {prior }}$ is written as

$$
\boldsymbol{\eta}^{\text {prior }}\left(\theta^{\prime}, \theta\right)=\sum_{\alpha=1}^{N} \mathbf{Y}^{\alpha, \text { prior }}\left(\theta^{\prime}\right) \Psi_{\alpha}(\boldsymbol{\Xi}(\theta))
$$

The realization $\mathrm{Y}^{\text {prior }}\left(\theta^{\prime}\right)$ of $\mathrm{Y}^{\text {prior }}$ is given by

$$
\mathrm{Y}^{\text {prior }}\left(\theta^{\prime}\right)=\left(\mathbf{Y}^{1, \text { prior }}\left(\theta^{\prime}\right), \ldots, \mathbf{Y}^{N, \text { prior }}\left(\theta^{\prime}\right)\right) \text {. }
$$

\subsection{Probability distribution of the prior model}

Let $\varepsilon \geq 0$ be any given positive or null real number. The probability density function $\mathrm{Y} \mapsto p_{\mathrm{Y}}^{\text {prior }}(\mathrm{Y})$ on $\mathrm{R}^{n N}$ of the random vector $\mathrm{Y}^{\text {prior }}=\left(\mathbf{Y}^{1, \text { prior }}, \ldots, \mathbf{Y}^{N, \text { prior }}\right)$ is such that the random vectors $\mathbf{Y}^{1, \text { prior }}, \ldots, \mathbf{Y}^{N \text {,prior }}$ are mutually independent and such that,

$$
\mathbf{Y}^{\alpha, \text { prior }}=2 \varepsilon\left|\underline{\mathbf{y}}^{\alpha}\right| \mathrm{U}_{\alpha}+\underline{\mathbf{y}}^{\alpha}-\varepsilon\left|\underline{\mathbf{y}}^{\alpha}\right|
$$

in which $\left|\underline{\mathbf{y}}^{\alpha}\right|$ is the vector $\left(\left|\underline{y}_{1}^{\alpha}\right|, \ldots,\left|\underline{y}_{n}^{\alpha}\right|\right)$ where $\underline{\mathbf{y}}^{1}, \ldots, \underline{\mathbf{y}}^{N}$ are the $N$ known vectors in $\mathrm{R}^{n}$ calculated in Step 4. In Eq. (21), $\left\{\mathrm{U}_{1}, \ldots, \mathrm{U}_{N}\right\}$ is a family of independent uniform random variables on $[0,1]$, defined on $\left(\Theta^{\prime}, \mathcal{T}^{\prime}, \mathcal{P}^{\prime}\right)$. Consequently, the component $Y_{j}^{\alpha, \text { prior }}$ of $\mathbf{Y}^{\alpha, \text { prior }}$ is a uniform random variable, centered in $\underline{y}_{j}^{\alpha}$ and the support of its probability distribution is written as

$$
s_{j}^{\alpha}=\left[\underline{y}_{j}^{\alpha}-\varepsilon\left|\underline{y}_{j}^{\alpha}\right|, \underline{y}_{j}^{\alpha}+\varepsilon\left|\underline{y}_{j}^{\alpha}\right|\right] .
$$


It can then be deduced that $E\left\{\boldsymbol{\eta}^{\text {prior }}\right\}=0$ and the mean values of the random VVC are such that

$$
E\left\{\mathbf{Y}^{\alpha, \text { prior }}\right\}=\underline{\mathbf{y}}^{\alpha} \quad, \quad \text { for } \alpha=1, \ldots, N,
$$

The statistical fluctuations of $\mathbf{Y}^{\alpha, \text { prior }}$ around the mean value $\mathbf{y}^{\alpha}$ is controlled by parameter $\varepsilon$. If $\varepsilon=0$, then $\mathbf{Y}^{\alpha, \text { prior }}=\underline{\mathbf{y}}^{\alpha}$ almost surely (deterministic case for the VVC of the PCE introduced in Step 4).

\subsection{Subset of independent realizations for the prior model}

Let $\left\{\left[\AA^{\text {prior }}\left(\mathbf{x}^{1}\right)\right], \ldots,\left[\AA^{\text {prior }}\left(\mathbf{x}^{N_{p}}\right)\right]\right\}$ be the $N_{p}$ random matrices associated with the prior model $V^{\text {prior. }}$. In Section 2-(2), we have seen that the matrix-valued random field $\{[\mathrm{A}(\mathbf{x})], \mathbf{x} \in \Omega\}$ must generally satisfy mathematical properties for that the stochastic boundary value problem has a unique stochastic solution verifying given properties. Let us assume that such mathematical properties are described as follows: The family of random matrices $\left\{\left[\mathrm{A}^{\text {prior }}\left(\mathbf{x}^{1}\right)\right], \ldots,\left[\mathrm{A}^{\text {prior }}\left(\mathbf{x}^{N_{p}}\right)\right]\right\}$ verifies, almost surely, a property denoted as $\mathcal{P}_{\text {rop }}$ (for instance, for all $k$ in $\left\{1, \ldots, N_{p}\right\}$, the random matrix $\left[\mathrm{A}^{\text {prior }}\left(\mathbf{x}^{k}\right)\right]$ should be positive define almost surely). By construction (see Section 2), for $\varepsilon=0$, property $\mathcal{P}_{\text {rop }}$ is verified almost surely. However, for $\varepsilon>0$, such a property can be not verified for certain realizations. Consequently, the rejection method is used to construct the subset of independent realizations for which $\mathcal{P}_{\text {rop }}$ is satisfied almost surely.

Let $\varepsilon$ be fixed (not equal to zero). Let $\mathrm{Y}^{\text {prior }}\left(\theta_{1}^{\prime}\right), \ldots, \mathrm{Y}^{\text {prior }}\left(\theta_{\nu^{\prime}}^{\prime}\right)$ be $\nu^{\prime}$ independent realizations of $\mathrm{Y}^{\text {prior }}$ for $\theta_{1}^{\prime}, \ldots, \theta_{\nu^{\prime}}^{\prime}$ in $\Theta^{\prime}$. Let $\boldsymbol{\Xi}\left(\theta_{1}\right), \ldots, \boldsymbol{\Xi}\left(\theta_{\nu}\right)$ be the $\nu$ independent realizations of $\boldsymbol{\Xi}$ (for $\theta_{1}, \ldots, \theta_{\nu}$ in $\Theta$ ) introduced in Step 4 of Section 3. For given $\theta_{\ell^{\prime}}^{\prime}$ and $\theta_{\ell}$, let $\mathrm{V}^{\text {prior }}\left(\theta_{\ell^{\prime}}^{\prime}, \theta_{\ell}\right)$ be the realization of $\mathrm{V}^{\text {prior }}$ and let $\left[\mathrm{A}^{\text {prior }}\left(\mathbf{x}^{1} ; \theta_{\ell^{\prime}}^{\prime}, \theta_{\ell}\right)\right], \ldots,\left[\mathrm{A}^{\text {prior }}\left(\mathbf{x}^{N_{p}} ; \theta_{\ell^{\prime}}^{\prime}, \theta_{\ell}\right)\right]$ be the corresponding realizations of $\left[\mathrm{A}^{\text {prior }}\left(\mathbf{x}^{1}\right)\right], \ldots,\left[\mathrm{A}^{\text {prior }}\left(\mathbf{x}^{N_{p}}\right)\right]$. Consequently, if the family $\left\{\left[\mathrm{A}^{\text {prior }}\left(\mathbf{x}^{1} ; \theta_{\ell^{\prime}}^{\prime}, \theta_{\ell}\right)\right], \ldots\right.$, $\left.\left[\mathrm{A}^{\text {prior }}\left(\mathbf{x}^{N_{p}} ; \theta_{\ell^{\prime}}^{\prime}, \theta_{\ell}\right)\right]\right\}$ verifies property $\mathcal{P}_{\text {rop }}$, then realization $\left(\theta_{\ell^{\prime}}^{\prime}, \theta_{\ell}\right)$ will be kept and, if not, this realization will be rejected. For fixed $\theta_{\ell^{\prime}}^{\prime}$, we then introduce the subset $\left\{\theta_{\ell_{1}}, \ldots, \theta_{\ell_{\widetilde{\nu}\left(\ell^{\prime}\right)}}\right\}$ of $\left\{\theta_{1}, \ldots, \theta_{\nu}\right\}$, with $\widetilde{\nu}\left(\ell^{\prime}\right) \leq \nu$, for which property $\mathcal{P}_{\text {rop }}$ is verified.

It should be noted that $\varepsilon$ will arbitrarily be fixed in the context of the use of the Bayesian method to construct the posterior model. In general, more $\varepsilon$ will be chosen large, more $\widetilde{\nu}\left(\ell^{\prime}\right)$ will be small. Therefore, a compromise will have to be chosen between the number $\widetilde{\nu}\left(\ell^{\prime}\right)$ of realizations to get convergence of the statistical estimators and a large value of $\varepsilon$ allowing large deviations from the prior model to be generated. 


\subsection{Prior probability density functions of the responses}

For all $\ell^{\prime}$ fixed in $\left\{1, \ldots, \nu^{\prime}\right\}$, the realizations $\mathbf{U}^{\text {prior }}\left(\theta_{\ell^{\prime}}^{\prime}, \theta_{\ell_{j}}\right)$ for $j=1, \ldots, \widetilde{\nu}\left(\ell^{\prime}\right)$ of the prior random vector $\mathbf{U}^{\text {prior }}=\left(\mathbf{U}^{\text {obs,prior }}, \mathbf{U}^{\text {nobs,prior }}\right)=\mathbf{h}\left(V^{\text {prior }}\right)$ are calculated with the stochastic computational model for the prior model $Y^{\text {prior }}$ of $Y$. For $1 \leq$ $k \leq m_{\mathrm{obs}}, U_{k}^{\text {prior }}$ is a component of the random observation vector $\mathbf{U}^{\text {obs,prior }}$ while, if $m_{\mathrm{obs}}+1 \leq k \leq m$, then $U_{k}^{\text {prior }}$ represents a component of the random vector $\mathbf{U}^{\text {nobs,prior }}$. The probability density function $u_{k} \mapsto p_{U_{k}^{\text {prior }}}\left(u_{k}\right)$ on $\mathrm{R}$ of the prior random variable $U_{k}^{\text {prior }}$ is then estimated using the above independent realizations and the kernel density estimation method [6].

\section{Posterior probability model of the random VVC using the classical Bayesian approach}

In this section, we present the use of the classical Bayesian approach to construct the posterior probability model $Y^{\text {post }}$ of the random VVC for which the prior probability model $Y^{\text {prior }}$ has been constructed in Section 5 .

\subsection{Conditional probability of the vector-valued random observation if VVC are given}

For a given vector $\mathrm{y}=\left(\mathbf{y}^{1}, \ldots, \mathbf{y}^{N}\right)$ in $\mathrm{R}^{n N}=\mathrm{R}^{n} \times \ldots \times \mathrm{R}^{n}$, let $\mathbf{U}=\left(\mathbf{U}^{\text {obs }}, \mathbf{U}^{\text {nobs }}\right)$ be the random vector with values in $\mathrm{R}^{m}=\mathrm{R}^{m_{\mathrm{obs}}} \times \mathrm{R}^{m_{\text {nobs }}}$, such that $\mathbf{U}=\mathbf{h}(\mathrm{V})$ (see Eq. (1)) in which the random vector $\mathrm{V}$ with values in $\mathrm{R}^{m_{\vee}}$ is given by $\mathrm{V}=\underline{\mathrm{V}}+$ $\sum_{j=1}^{n} \sqrt{\lambda_{j}} \eta_{j} \mathrm{~W}^{j}$ (see Eq. (15)) and for which the random vector $\boldsymbol{\eta}=\left(\eta_{1}, \ldots, \eta_{n}\right)$ with values in $\mathrm{R}^{n}$ is given by $\boldsymbol{\eta}=\sum_{\alpha=1}^{N} \mathbf{y}^{\alpha} \Psi_{\alpha}(\boldsymbol{\Xi})$ (see Eq. (16)).

We introduce the conditional probability density function $\mathbf{u}^{\text {obs }} \mapsto p_{\mathbf{U}^{\text {obs }} \mid \mathrm{Y}}\left(\mathbf{u}^{\text {obs }} \mid \mathrm{Y}\right)$ (defined on $\mathrm{R}^{m_{\mathrm{obs}}}$ and with respect to the Lebesgue measure $d \mathbf{u}^{\mathrm{obs}}=d u_{1}^{\mathrm{obs}} \ldots d u_{m_{\mathrm{obs}}}^{\mathrm{obs}}$ ) of random observation vector $\mathbf{U}^{\text {obs }}$ if $\mathrm{Y}=\left(\mathbf{Y}^{1}, \ldots, \mathbf{Y}^{N}\right)$ is equal to the given vector $\mathrm{y}=\left(\mathbf{y}^{1}, \ldots, \mathbf{y}^{N}\right)$ in $\mathrm{R}^{n N}$.

Consequently, the random observation vector $\mathbf{U}^{\text {obs }}=\left(U_{1}^{\text {obs }}, \ldots, U_{m_{\text {obs }}}^{\text {obs }}\right)$ depends on $\mathrm{Y}=\mathrm{Y}$ and the stochastic computational model allows the conditional probability density functions $\mathbf{u}^{\mathrm{obs}} \mapsto p_{\mathbf{U}^{\mathrm{obs}} \mid \mathrm{Y}}\left(\mathbf{u}^{\mathrm{obs}} \mid \mathrm{Y}\right)$ and $u_{k}^{\mathrm{obs}} \mapsto p_{U_{k}^{\mathrm{obs}} \mid \mathrm{Y}}\left(u_{k}^{\mathrm{obs}} \mid \mathrm{Y}\right)$ to be calculated. 


\subsection{Formulation using the Bayesian method}

The posterior random vector $\mathbf{U}^{\text {post }}=\left(\mathbf{U}^{\text {obs,post }}, \mathbf{U}^{\text {nobs,post }}\right)$ with values in $\mathrm{R}^{m}=$ $\mathrm{R}^{m_{\text {obs }}} \times \mathrm{R}^{m_{\text {nobs }}}$ is written (see Eq. (1)) as

$$
\mathbf{U}^{\text {post }}=\mathbf{h}\left(\mathrm{V}^{\text {post }}\right), \mathbf{U}^{\text {obs,post }}=\mathbf{h}^{\text {obs }}\left(\mathrm{V}^{\text {post }}\right), \mathbf{U}^{\text {nobs,post }}=\mathbf{h}^{\text {nobs }}\left(\mathrm{V}^{\text {post }}\right),
$$

in which the $\mathrm{R}^{m_{\vee}}$-valued random vector $\mathrm{V}^{\text {post }}$ is the posterior model of $\mathrm{V}^{\text {prior }}$. Taking into account Eqs. (15) to (17), the posterior model of $\mathrm{V}^{\text {prior }}$ is written as

$$
\mathrm{V}^{\text {post }}=\underline{\mathrm{V}}+\sum_{j=1}^{n} \sqrt{\lambda_{j}} \eta_{j}^{\text {post }} \mathrm{W}^{j},
$$

in which the posterior stochastic model $\boldsymbol{\eta}^{\text {post }}=\left(\eta_{1}^{\text {post }}, \ldots, \eta_{n}^{\text {post }}\right)$ is a $\mathrm{R}^{n}$-valued random variable defined on $\left(\Theta^{\prime} \times \Theta, \mathcal{T}^{\prime} \otimes \mathcal{T}, \mathcal{P}^{\prime} \otimes \mathcal{P}\right)$, such that

$$
\boldsymbol{\eta}^{\text {post }}=\sum_{\alpha=1}^{N} \mathbf{Y}^{\alpha, \text { post }} \Psi_{\alpha}(\boldsymbol{\Xi})
$$

in which the family of $\mathrm{R}^{n}$-valued random variables $\left\{\mathbf{Y}^{1, \text { post }}, \ldots, \mathbf{Y}^{N \text {,post }}\right\}$ are defined on the probability space $\left(\Theta^{\prime}, \mathcal{T}^{\prime}, \mathcal{P}^{\prime}\right)$. As previously, we introduce the $\mathrm{R}^{n N_{\text {}}}$ valued random vector $Y^{\text {post }}$ defined on $\left(\Theta^{\prime}, \mathcal{T}^{\prime}, \mathcal{P}^{\prime}\right)$ such that

$$
\mathrm{Y}^{\text {post }}=\left(\mathbf{Y}^{1, \text { post }}, \ldots, \mathbf{Y}^{N, \text { post }}\right)
$$

whose its probability distribution is represented by the probability density function $\mathrm{y} \mapsto p_{\mathrm{Y}}^{\text {post }}(\mathrm{Y})$ on $\mathrm{R}^{n N}$ with respect to the Lebesgue measure $d \mathrm{y}=d \mathrm{y}_{1} \ldots d \mathrm{y}_{n m}$.

Let $\mathbf{u}^{\exp , 1}, \ldots, \mathbf{u}^{\exp , \nu_{\exp }}$ be the $\nu_{\exp }$ independent experimental data $\mathbf{U}^{\exp }$ corresponding to observation vector $\mathbf{U}^{\text {obs }}$. The Bayesian method (see for instance $[4,5,7$, $9,40,50,51,54])$ allows the posterior probability density function $p_{\mathrm{Y}}^{\text {post }}(\mathrm{y})$ to be calculated, using the prior probability density function $p_{\mathrm{Y}}^{\text {prior }}(\mathrm{y})$ and using the experimental values $p_{\mathbf{U}^{\text {obs }} \mid \mathrm{Y}}\left(\mathbf{u}^{\text {exp }, \ell} \mid \mathrm{Y}\right)$ of the conditional probability density function $p_{\mathbf{U}^{\text {obs } \mid Y}}\left(\mathbf{u}^{\text {obs }} \mid \mathrm{Y}\right)$, as

$$
p_{\mathrm{Y}}^{\text {post }}(\mathrm{y})=L^{\text {bayes }}(\mathrm{y}) p_{\mathrm{Y}}^{\text {prior }}(\mathrm{y})
$$

in which $\mathrm{Y} \mapsto L^{\text {bayes }}(\mathrm{Y})$ is the likelihood function defined on $\mathrm{R}^{n N}$, with values in $\mathrm{R}^{+}$, such that

$$
L^{\text {bayes }}(\mathrm{Y})=\frac{\Pi_{\ell=1}^{\nu \text { exp }} p_{\mathbf{U}^{\text {obs }} \mid \mathrm{Y}}\left(\mathbf{u}^{\text {exp }, \ell} \mid \mathrm{Y}\right)}{E\left\{\Pi_{\ell=1}^{\nu \exp } p_{\mathbf{U}^{\text {obs }} \mid \mathrm{Y}}\left(\mathbf{u}^{\text {exp }, \ell} \mid Y^{\text {prior }}\right)\right\}} .
$$


Eq. (29) shows that likelihood function $L^{\text {bayes }}$ must verify the following equation,

$$
E\left\{L^{\text {bayes }}\left(\mathrm{Y}^{\text {prior }}\right)\right\}=\int_{\mathrm{R}^{n N}} L^{\text {bayes }}(\mathrm{Y}) p_{\mathrm{Y}}^{\text {prior }}(\mathrm{Y}) d \mathrm{Y}=1 .
$$

\subsection{Posterior probability density functions of the responses}

The probability density function $\mathbf{u} \mapsto p_{\mathbf{U}^{\text {post }}}(\mathbf{u})$ on $\mathrm{R}^{m}$ of the posterior random vector $\mathbf{U}^{\text {post }}$ is then given by $p_{\mathbf{U}^{\text {post }}}(\mathbf{u})=\int_{\mathrm{R}^{n N}} p_{\mathbf{U} \mid \mathrm{Y}}(\mathbf{u} \mid \mathrm{Y}) p_{\mathrm{Y}}^{\text {post }}(\mathrm{Y}) d \mathrm{Y}$ in which $p_{\mathbf{U} \mid \mathrm{Y}}(\mathbf{u} \mid \mathrm{Y})$ is the conditional probability density function of $\mathbf{U}$ given $\mathrm{Y}=\mathrm{y}$ and which is constructed using the computational model defined in Section 6.1. Using Eq. (28), this last equation can be rewritten as $p_{\mathbf{U}^{\text {post }}}(\mathbf{u})=E\left\{L^{\text {bayes }}\left(\mathrm{Y}^{\text {prior }}\right) p_{\mathbf{U} \mid \mathbf{Y}}\left(\mathbf{u} \mid Y^{\text {prior }}\right)\right\}$. Let $U_{k}^{\text {post }}$ be any component of random vector $\mathbf{U}^{\text {post }}$. For $1 \leq k \leq m_{\mathrm{obs}}, U_{k}^{\text {post }}$ represents a component of random observation vector $\mathbf{U}^{\text {obs,post }}$ while, if $m_{\mathrm{obs}}+1 \leq$ $k \leq m$, then $U_{k}^{\text {post }}$ represents a component of random vector $\mathbf{U}^{\text {nobs,post }}$. Consequently, the probability density function $u_{k} \mapsto p_{U_{k}^{\text {post }}}\left(u_{k}\right)$ on $\mathrm{R}$ of the posterior random variable $U_{k}^{\text {post }}$ is then given by

$$
p_{U_{k}^{\text {post }}}\left(u_{k}\right)=E\left\{L^{\text {bayes }}\left(\mathrm{Y}^{\text {prior }}\right) p_{U_{k} \mid \mathrm{Y}}\left(u_{k} \mid \mathrm{Y}^{\text {prior }}\right)\right\},
$$

in which $p_{U_{k} \mid \mathrm{Y}}\left(u_{k} \mid \mathrm{Y}\right)$ is the conditional probability density function of the real valued random variable $U_{k}$ given $\mathrm{Y}=\mathrm{y}$ and which is constructed using the computational model defined in Section 6.1.

\subsection{Computational aspects}

We use the notation introduced in Section 5.4 concerning the realizations of $Y$ and $\Xi$. For $\nu^{\prime}$ sufficiently large, the right-hand side of Eq. (31) can be estimated by

$$
p_{U_{k}^{\text {post }}}\left(u_{k}\right) \simeq \frac{1}{\nu^{\prime}} \sum_{\ell^{\prime}=1}^{\nu^{\prime}} L^{\text {bayes }}\left(\mathrm{Y}^{\text {prior }}\left(\theta_{\ell^{\prime}}^{\prime}\right)\right) p_{U_{k} \mid \mathrm{Y}}\left(u_{k} \mid \mathrm{Y}^{\text {prior }}\left(\theta_{\ell^{\prime}}^{\prime}\right)\right),
$$

For fixed $\theta_{\ell^{\prime}}^{\prime}$, the computational model defined in Section 6.1 is used to calculate the $\widetilde{\nu}\left(\ell^{\prime}\right)$ independent realizations $\mathbf{U}\left(\theta_{\ell_{1}} \mid Y^{\text {prior }}\left(\theta_{\ell^{\prime}}^{\prime}\right)\right), \ldots, \mathbf{U}\left(\theta_{\ell_{\widetilde{\nu}\left(\ell^{\prime}\right)}} \mid Y^{\text {prior }}\left(\theta_{\ell^{\prime}}^{\prime}\right)\right)$ for $\mathrm{Y}=\mathrm{Y}^{\text {prior }}\left(\theta_{\ell^{\prime}}^{\prime}\right)$. We can then deduce $\mathbf{U}^{\text {obs }}\left(\theta_{\ell_{1}} \mid \mathrm{Y}^{\text {prior }}\left(\theta_{\ell^{\prime}}^{\prime}\right)\right), \ldots, \mathbf{U}^{\text {obs }}\left(\theta_{\ell_{\tilde{\nu}\left(\ell^{\prime}\right)}} \mid Y^{\text {prior }}\left(\theta_{\ell^{\prime}}^{\prime}\right)\right)$ and, for all fixed $k, U_{k}\left(\theta_{\ell_{1}} \mid \mathrm{Y}^{\text {prior }}\left(\theta_{\ell^{\prime}}^{\prime}\right)\right), \ldots, U_{k}\left(\theta_{\ell_{\widetilde{\nu}\left(\ell^{\prime}\right)}} \mid Y^{\text {prior }}\left(\theta_{\ell^{\prime}}^{\prime}\right)\right)$.

(1) Using the independent realizations $\mathbf{U}^{\text {obs }}\left(\theta_{\ell_{1}} \mid \mathrm{Y}^{\text {prior }}\left(\theta_{\ell^{\prime}}^{\prime}\right)\right), \ldots, \mathbf{U}^{\text {obs }}\left(\theta_{\ell_{\tilde{\nu}\left(\ell^{\prime}\right)}} \mid Y^{\text {prior }}\left(\theta_{\ell^{\prime}}^{\prime}\right)\right)$ and the multivariate Gaussian kernel density estimation (see Appendix A), we can estimate $p_{\mathbf{U}^{\text {obs }} \mid Y}\left(\left.\mathbf{u}^{\text {exp }, \ell}\right|_{Y^{\text {prior }}}\left(\theta_{\ell^{\prime}}^{\prime}\right)\right)$ and then, using Eq. (29), we can compute $L^{\text {bayes }}\left(\mathrm{Y}^{\text {prior }}\left(\theta_{\ell^{\prime}}^{\prime}\right)\right)$ for $\ell^{\prime}=1, \ldots, \nu^{\prime}$. 
(2) Using the independent realizations $U_{k}\left(\theta_{\ell_{1}} \mid \mathrm{Y}^{\text {prior }}\left(\theta_{\ell^{\prime}}^{\prime}\right)\right), \ldots, U_{k}\left(\theta_{\ell_{\tilde{\nu}\left(\ell^{\prime}\right)}} \mid Y^{\text {prior }}\left(\theta_{\ell^{\prime}}^{\prime}\right)\right)$ and the kernel estimation method [6], we can estimate $p_{U_{k} \mid \mathrm{Y}}\left(u_{k} \mid \mathrm{Y}^{\text {prior }}\left(\theta_{\ell^{\prime}}^{\prime}\right)\right)$ and then, using Eq. (32), we can estimate $p_{U_{k}^{\text {post }}}\left(u_{k}\right)$.

\section{Posterior probability model of the random VVC using a new approach derived from the Bayesian approach}

In Section 6, we have presented the classical Bayesian approach to construct the posterior model $\mathrm{Y}^{\text {post }}$ of the prior model $\mathrm{Y}^{\text {prior }}$ of the random VVC. Nevertheless, in the application presented in Section 8, we will see that, for a very high-dimension problem (the random vector $Y^{\text {post }}$ has several millions of components), the usual Bayesian method can be improved to get a more efficient method derived from the classical one and presented below.

It should be noted that Eq. (31) can be rewritten as

$$
p_{U_{k}^{\text {post }}}\left(u_{k}\right)=E\left\{L^{1 \mathrm{ls}} p_{U_{k} \mid \mathrm{Y}}\left(u_{k} \mid \mathrm{Y}^{\text {prior }}\right)\right\},
$$

in which the positive-valued random variable $L^{\text {lls }}$ defined on $\left(\Theta^{\prime}, \mathcal{T}^{\prime}, \mathcal{P}^{\prime}\right)$ is such that $L^{\text {lls }}=L^{\text {bayes }}\left(\mathrm{Y}^{\text {prior }}\right)$ and such that $E\left\{L^{\text {lls }}\right\}=1$ (see Eq. (30)). The $\nu^{\prime}$ independent realizations of $L^{\mathrm{lls}}$ are $L^{\mathrm{lls}}\left(\theta_{1}^{\prime}\right), \ldots, L^{\mathrm{lls}}\left(\theta_{\nu^{\prime}}^{\prime}\right)$ such that $L^{\mathrm{lls}}\left(\theta_{\ell^{\prime}}^{\prime}\right)=$ $L^{\text {bayes }}\left(Y^{\text {prior }}\left(\theta_{\ell^{\prime}}^{\prime}\right)\right)$. With such a notation, Eq. (32) can be rewritten as

$$
p_{U_{k}^{\text {post }}}\left(u_{k}\right) \simeq \frac{1}{\nu^{\prime}} \sum_{\ell^{\prime}=1}^{\nu^{\prime}} L^{1 \mathrm{ls}}\left(\theta_{\ell^{\prime}}^{\prime}\right) p_{U_{k} \mid \mathrm{Y}}\left(u_{k} \mid \mathrm{Y}^{\text {prior }}\left(\theta_{\ell^{\prime}}^{\prime}\right)\right),
$$

and $E\left\{L^{1 \mathrm{ls}}\right\}=1$ yields

$$
\frac{1}{\nu^{\prime}} \sum_{\ell^{\prime}=1}^{\nu^{\prime}} L^{11 \mathrm{~s}}\left(\theta_{\ell^{\prime}}^{\prime}\right) \simeq 1
$$

The method proposed consists in using Eq. (34), but in replacing $L^{\text {1ls }}=L^{\text {bayes }}\left(\mathrm{Y}^{\text {prior }}\right)$ by another random variable for which the vector $\mathbf{L}^{\text {1ls }}=\left(L^{\text {lls }}\left(\theta_{1}^{\prime}\right), \ldots, L^{1 \mathrm{ls}}\left(\theta_{\nu^{\prime}}^{\prime}\right)\right)$ of the realizations of $L^{\text {1ls }}$ is constructed as the unique solution of the following linear least square optimization problem with nonnegativity constraints,

$$
\mathbf{L}^{\text {1ls }}=\arg \min _{\mathbf{L} \in \mathcal{G}_{\mathrm{ad}}} G(\mathbf{L})
$$


The admissible set $\mathcal{G}_{\text {ad }}$ is defined as

$$
\mathcal{G}_{\mathrm{ad}}=\left\{\mathbf{L}=\left(L_{1}, \ldots, L_{\nu^{\prime}}\right) \in \mathrm{R}^{\nu^{\prime}} ; L_{1} \geq 0, \ldots, L_{\nu^{\prime}} \geq 0 ; \frac{1}{\nu^{\prime}} \sum_{\ell^{\prime}=1}^{\nu^{\prime}} L_{\ell^{\prime}}=1\right\}
$$

The cost function $\mathbf{L} \mapsto G(\mathbf{L})$ is defined as

$$
G(\mathbf{L})=\sum_{k=1}^{m_{\mathrm{obs}}} \int_{\mathrm{R}}\left(\widehat{p}_{U_{k}^{\mathrm{exp}}}\left(u_{k}^{\mathrm{obs}}\right)-\frac{1}{\nu^{\prime}} \sum_{\ell^{\prime}=1}^{\nu^{\prime}} L_{\ell^{\prime}} p_{U_{k}^{\mathrm{obs}} \mid \mathrm{Y}}\left(u_{k}^{\mathrm{obs}} \mid \mathrm{Y}^{\text {prior }}\left(\theta_{\ell^{\prime}}^{\prime}\right)\right)\right)^{2} d u_{k}^{\mathrm{obs}}
$$

in which $u_{k}^{\text {obs }} \mapsto \widehat{p}_{U_{k}^{\exp }}\left(u_{k}^{\mathrm{obs}}\right)$ is an estimation of the probability density function of the random variable $U_{k}^{\exp }$ carried out with the experimental data $u_{k}^{\exp , 1}, \ldots, u_{k}^{\exp , \nu_{\exp }}$ (see Section 2-(3)) and using the kernel estimation method [6]. In Eq. (38), $p_{U_{k}^{\text {obs } \mid Y}}\left(u_{k}^{\text {obs }} \mid Y^{\text {prior }}\left(\theta_{\ell^{\prime}}^{\prime}\right)\right)$ is estimated as explained in Section 6.4. The optimization problem defined by Eq. (36) can be solved, for instance, using the algorithm described in [26]. The quality assessment is performed using Eq. (34) for $k$ such that $m_{\mathrm{obs}}+1 \leq k \leq m$, that is to say, when $U_{k}^{\text {post }}$ represents a component of the random vector $\mathbf{U}^{\text {nobs,post }}$ which is not observed and which is then not used in Eq. (36) for the calculation of $\mathbf{L}^{11 \mathrm{~s}}$.

In theory, the Bayesian approach presented in Section 6 can be used in high dimension and for a few experimental data (small value of $\nu_{\exp }$ ), but in practice, for the high-dimension case (very large value of the product $n N$ such as several millions), the posterior probability model significantly improves the prior model if many experimental data are available (large value of $\nu_{\text {exp }}$ ). On the other hand, the method proposed in Section 7 requires the estimation of the probability density function of the experimental observations $U_{k}^{\exp }$ (using the kernel density estimation in the context of nonparametric statistics). Such an estimation is not correct if $\nu_{\exp }$ is too small and must be sufficiently large (for instance $\nu_{\exp } \simeq 100$ ). In Section 8 which is devoted to the application, for $n N=550 \times 10625=5843750$ (high-dimension case), $m_{\text {obs }}=50$ while $m=1017$ (partial data) and $\nu_{\text {exp }}=200$ (limited data), we will see that the posterior model constructed with the method proposed in Section 7 is more efficient than the Bayesian method presented in Section 6. Finally, it should be noted that, as soon as the independent realizations $\mathrm{Y}^{\text {prior }}\left(\theta_{1}^{\prime}\right), \ldots, \mathrm{Y}^{\text {prior }}\left(\theta_{\nu^{\prime}}^{\prime}\right)$ are given, Eqs. (36) to (38) correspond to the generator of random variable $L^{\text {lls }}$ allowing the realizations $L^{1 \mathrm{ls}}\left(\theta_{1}^{\prime}\right), \ldots, L^{\text {1ls }}\left(\theta_{\nu^{\prime}}^{\prime}\right)$ to be generated. For any $\nu^{\prime}$ and for any realizations of $Y^{\text {prior }}$, we then have a generator of 
realizations of $L^{11 s}$. For any given measurable mapping $\mathbf{g}$ defined on $\mathrm{R}^{m}$, we can then compute the quantity

$$
E\left\{\mathbf{g}\left(\mathbf{U}^{\text {post }}\right)\right\} \simeq \frac{1}{\nu^{\prime}} \sum_{\ell^{\prime}=1}^{\nu^{\prime}} \frac{L^{1 \mathrm{ls}}\left(\theta_{\ell^{\prime}}^{\prime}\right)}{\widetilde{\nu}\left(\ell^{\prime}\right)} \sum_{j=1}^{\widetilde{\nu}\left(\ell^{\prime}\right)} \mathbf{g}\left(\mathbf{U}^{\text {prior }}\left(\theta_{\ell^{\prime}}^{\prime}, \theta_{\ell_{j}}\right)\right)
$$

where $\mathbf{U}^{\text {prior }}\left(\theta_{\ell^{\prime}}^{\prime}, \theta_{\ell_{j}}\right)=\mathbf{h}\left(\mathrm{V}^{\text {prior }}\left(\theta_{\ell^{\prime}}^{\prime}, \theta_{\ell_{j}}\right)\right)$ is calculated with the computational model and where

$$
\left.\mathrm{V}^{\text {prior }}\left(\theta_{\ell^{\prime}}^{\prime}, \theta_{\ell_{j}}\right)\right)=\underline{\mathrm{V}}+\sum_{j=1}^{n} \sqrt{\lambda_{j}} \eta_{j}^{\text {prior }}\left(\theta_{\ell^{\prime}}^{\prime}, \theta_{\ell_{j}}\right) \mathrm{w}^{j},
$$

with

$$
\boldsymbol{\eta}^{\text {prior }}\left(\theta_{\ell^{\prime}}^{\prime}, \theta_{\ell_{j}}\right)=\sum_{\alpha=1}^{N} \mathbf{Y}^{\alpha, \text { prior }}\left(\theta_{\ell^{\prime}}^{\prime}\right) \Psi_{\alpha}\left(\boldsymbol{\Xi}\left(\theta_{\ell_{j}}\right)\right)
$$

\section{Application}

In this work, we reuse the example introduced in [48], but the experimental data are different. The stochastic model used to generate the experimental data (numerical experiments) strongly differs from the family of prior algebraic stochastic models (PASM). This means that the prior stochastic model cannot fit the experiments and consequently, the posterior model must be constructed using the experimental data. Consequently, the first four steps of the methodology must be redone and will then be presented. We will present the identification of the posterior model using the two approaches presented in Sections 6 and 7. In order to give readability to the present paper, we give again some explanations, already introduced in [48], but required to obtain a good understanding.

\subsection{Definition of the stochastic boundary value problem at the meso-scale}

We consider a microstructure represented by the domain $\Omega=(] 0,1[)^{3}$ of $\mathrm{R}^{3}$ with generic point $\mathbf{x}=\left(x_{1}, x_{2}, x_{3}\right)$ (see Fig. 1). Domain $\Omega$ is occupied by a heterogeneous complex material modeled by a statistically homogeneous and anisotropic elastic random medium at the meso-scale. For this meso-scale modeling, the elastic properties of the microstructure are then defined by the non-Gaussian fourthorder tensor-valued random field $\{\mathrm{C}(\mathbf{x}), \mathbf{x} \in \Omega\}$ in which $\mathrm{C}(\mathbf{x})=\left\{\mathrm{C}_{i j k \ell}(\mathbf{x})\right\}_{i j k \ell}$. Let $\left\{\mathbf{u}(\mathbf{x})=\left(u_{1}(\mathbf{x}), u_{2}(\mathbf{x}), u_{3}(\mathbf{x})\right), \mathbf{x} \in \Omega\right\}$ be the displacement random field at the meso-scale. The random constitutive equation is then written as $\mathbf{s}_{j k}(\mathbf{x})=$ 


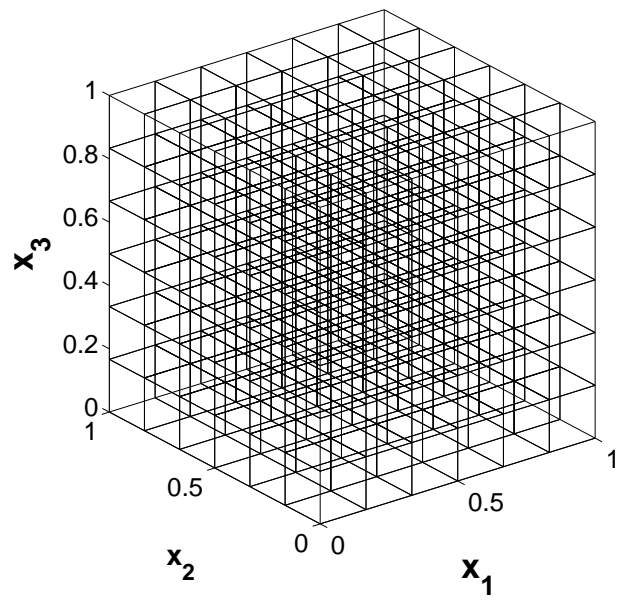

Figure 1: Finite element mesh of the domain.

$\mathrm{C}_{j k \ell m}(\mathbf{x}) \varepsilon_{\ell m}(\mathbf{x})$, in which $\mathbf{s}$ is the stress tensor and $\varepsilon$ is the strain tensor such that $\varepsilon_{\ell m}(\mathbf{u}(\mathbf{x}))=\frac{1}{2}\left(\partial u_{\ell}(\mathbf{x}) / \partial x_{m}+\partial u_{m}(\mathbf{x}) / \partial x_{\ell}\right)$. The boundary $\partial \Omega$ is written as $\Gamma_{0} \cup \Gamma_{\text {obs }} \cup \Gamma$. A Dirichlet condition $\mathbf{u}=0$ is given on $\Gamma_{0}$ while a Neumann condition is given on $\Gamma$ corresponding to the application of a given deterministic surface force field $\mathbf{g}^{\Gamma}(\mathbf{x})=\left(g_{1}^{\Gamma}(\mathbf{x}), g_{2}^{\Gamma}(\mathbf{x}), g_{3}^{\Gamma}(\mathbf{x})\right)$. There is no surface force field applied to $\Gamma_{\text {obs }}$ which is the part of the boundary for which field $\mathbf{u}$ is observed (this corresponds to the hypothesis for which only partial experimental data are observed and then are available). The stochastic boundary value problem consists in finding the second-order random field $\{\mathbf{u}(\mathbf{x}), \mathbf{x} \in \Omega\}$ such that

$$
\begin{array}{rcl}
-\operatorname{div} \mathbf{s}=0 & \text { in } & \Omega, \\
\mathbf{u}(\mathbf{x})=\mathbf{0} & \text { on } & \Gamma_{0}, \\
\mathbf{s}(\mathbf{x}) \mathbf{n}(\mathbf{x})=\mathbf{g}^{\Gamma}(\mathbf{x}) \quad \text { on } \quad \Gamma & \text { and } & \mathbf{s}(\mathbf{x}) \mathbf{n}(\mathbf{x})=\mathbf{0} \text { on } \Gamma_{\text {obs }},
\end{array}
$$

in which $\mathbf{n}(\mathbf{x})=\left(n_{1}(\mathbf{x}), n_{2}(\mathbf{x}), n_{3}(\mathbf{x})\right)$ is the outward unit normal to $\partial \Omega$, where $\{\operatorname{div} \mathbf{s}(\mathbf{x})\}_{j}=\partial s_{j k}(\mathbf{x}) / \partial x_{k}$ and where the random constitutive equation is defined above.

8.2. Introduction of a family of prior algebraic stochastic models (PASM) for the random field $\{\mathrm{C}(\boldsymbol{x}), \boldsymbol{x} \in \Omega\}$

We apply Step 1 of the methodology presented in Section 3. The stochastic boundary value problem defined by Eq. (42) is elliptic. A family $\left\{\mathrm{C}^{\mathrm{PASM}}(\mathbf{x} ; \mathbf{w}), \mathbf{x} \in \Omega\right\}_{\mathbf{w}}$ 
of PASM is introduced to represent the fourth-order tensor-valued random field $\{\mathrm{C}(\mathbf{x}), \mathbf{x} \in \Omega\}$. For all $\mathbf{x}$ in $\Omega, \mathrm{C}^{\mathrm{PASM}}(\mathbf{x} ; \mathbf{w})$ is represented by the symmetric positive-definite $(6 \times 6)$ real random matrix $\left[\mathrm{A}^{\mathrm{PASM}}(\mathbf{x} ; \mathbf{w})\right]$ such that $\left[\mathrm{A}^{\mathrm{PASM}}(\mathbf{x} ; \mathbf{w})\right]_{I J}$ $=C_{i j k \ell}^{\text {PASM }}(\mathbf{x} ; \mathbf{w})$ and depends on the vector-valued parameter $\mathbf{w}$. Such a family $\left\{\left[\mathrm{A}^{\mathrm{PASM}}(\mathbf{x} ; \mathbf{w})\right], \mathbf{x} \in \Omega\right\}_{\mathbf{w}}$ of PASM cannot arbitrarily be chosen (see for instance $[2,3,56]$ for the scalar case). In this application, we are interested in the anisotropic case (tensor case) and we then propose to choose the stochastic model as follows. It is assumed that the mean value $[\underline{\mathrm{A}}]=E\left\{\left[\mathrm{~A}^{\mathrm{PASM}}(\mathbf{x} ; \mathbf{w})\right]\right\}$ is independent of $\mathbf{x}$ and is a positive-definite matrix. For all $\mathbf{x}$ in $\Omega,\left[\mathrm{A}^{\mathrm{PASM}}(\mathbf{x} ; \mathbf{w})\right]$ is written as $\left[\mathrm{A}^{\mathrm{PASM}}(\mathbf{x} ; \mathbf{w})\right]=\left[A^{0}\right]+\left[\mathbf{A}^{\mathrm{PASM}}(\mathbf{x} ; \mathbf{w})\right]$, in which the positive-definite matrix $\left[A^{0}\right]$ is written as $\left[A^{0}\right]=\varepsilon_{0}[\underline{\mathrm{A}}]$ with $0<\varepsilon_{0}<1$ (which can be chosen as small as one wants), and where $\left[\mathbf{A}^{\mathrm{PASM}}(\mathbf{x} ; \mathbf{w})\right]$ is a positive-definite random matrix. In this application, $\varepsilon_{0}$ is chosen to the value $10^{-6}$. The family of random fields $\left\{\left[\mathbf{A}^{\mathrm{PASM}}(\mathbf{x} ; \mathbf{w})\right], \mathbf{x} \in \Omega\right\}_{\mathbf{w}}$ is defined in $[43,44]$ which yields a stochastic nonuniform ellipticity condition. The family of random fields $\left\{\left[\mathrm{A}^{\mathrm{PASM}}(\mathbf{x} ; \mathbf{w})\right], \mathbf{x} \in\right.$ $\Omega\}_{\mathbf{w}}$, defined above, then yields a stochastic ellipticity condition which implies that the second-order random solution of the stochastic boundary value problem continuously depend from its parameters. It can then be deduced that the mean value $[\underline{A}]$ of $\left[\mathbf{A}^{\mathrm{PASM}}(\mathbf{x} ; \mathbf{w})\right]$ is such that $[\underline{A}]=E\left\{\left[\mathbf{A}^{\mathrm{PASM}}(\mathbf{x} ; \mathbf{w})\right]\right\}=[\underline{\mathrm{A}}]-\left[A^{0}\right]=$ $\left(1-\varepsilon_{0}\right)[\underline{\mathrm{A}}]$ which is then a positive-definite matrix. For this PASM, there exits a generator of independent realizations. The vector-valued parameter is $\mathbf{w}=$ $\left(\delta, L_{c}\right) \in \mathcal{C}_{\mathrm{ad}}$ in which $\delta>0$ is a real parameter controlling the level of statistical fluctuations of the field and $L_{c}>0$ is a correlation length controlling the spatial correlation of the field. In [43, 44], it is proven that, for all $\mathbf{x}$ in $\Omega$, $\left[\mathbf{A}^{\mathrm{PASM}}(\mathbf{x} ; \mathbf{w})\right]=[\underline{\mathcal{L}}]^{T}\left[\mathcal{L}\left(\mathbf{x} ; \delta, L_{c}\right)\right]^{T}\left[\mathcal{L}\left(\mathbf{x} ; \delta, L_{c}\right)\right][\underline{\mathcal{L}}]$ in which $[\underline{A}]=[\underline{\mathcal{L}}]^{T}[\underline{\mathcal{L}}]$ and where the random upper triangular matrix $\left[\mathcal{L}\left(\mathbf{x} ; \delta, L_{c}\right)\right]$ depends on $\mathbf{x}, \delta$ and $L_{c}$ and is such that $E\left\{\left[\mathcal{L}\left(\mathbf{x} ; \delta, L_{c}\right)\right]^{T}\left[\mathcal{L}\left(\mathbf{x} ; \delta, L_{c}\right)\right]\right\}=\left[I_{6}\right]$. As explained in [43, 44], the random upper triangular matrix $\left[\mathcal{L}\left(\mathbf{x} ; \delta, L_{c}\right)\right]$ is explicitly expressed as a function of 21 independent homogeneous normalized Gaussian real-valued random fields for which the 21 spatial autocorrelation functions depend on 63 real parameters $L_{1}^{j j^{\prime}}, L_{2}^{j j^{\prime}}, L_{3}^{j j^{\prime}}$ for $1 \leq j \leq j^{\prime} \leq 6$ which represent the spatial correlation lengths. In this application, we have used $L_{c}=L_{1}^{j j^{\prime}}=L_{2}^{j j^{\prime}}=L_{3}^{j j^{\prime}}$ for $1 \leq j \leq j^{\prime} \leq 6$. Consequently, the spatial correlation lengths of the random field $\left\{\left[\mathbf{A}^{\mathrm{PASM}}(\mathbf{x} ; \mathbf{w})\right], \mathbf{x} \in \Omega\right\}_{\mathbf{w}}$ is controlled by only one parameter which is $L_{c}$. 


\subsection{Stochastic finite element approximation of the stochastic boundary value problem}

The cube (] $0,1[)^{3}$ is meshed with $6 \times 6 \times 6=216$ finite elements using 8-nodes finite elements (see Fig. 1). There are 8 integration points in each finite element. We then have $N_{p}=1728$ integration points. The dimension of vector $\mathrm{V}^{\mathrm{PASM}}(\mathbf{w})$ discretizing $\left[\mathrm{A}^{\mathrm{PASM}}(. ; \mathbf{w})\right]$ is then $m_{\mathrm{V}}=21 \times N_{p}=36288$. Concerning the boundary conditions, the displacements are locked at points $(1,0,0),(1,1,0),(1,1,1)$ and $(1,0,1)$ corresponding to the 4 corners of the face of the cube in the plane $x_{1}=1$. An external point load $(0,1,0)$ is applied to the node of coordinates $(0,0,1)$. The observed degrees of freedom, for which there are available experimental data, are the $x_{2}$ - and $x_{3}$-displacements of the nodes located inside the face $x_{1}=0$. Since there are 49 nodes on each face of the cube whose 25 nodes inside the face, there are $m_{\mathrm{obs}}=2 \times 25=50$ observed degrees of freedom. The number of degrees of freedom for which no experimental data are available is $m_{\text {nobs }}=967$ and the total number of degrees of freedom is then $m=1017$.

\subsection{Experimental data sets}

The objective is the validation of the methodology proposed for partial and limited experimental data. The experimental data are then synthetically generated using the stochastic boundary value problem for which the elasticity tensor is a non-Gaussian elastic random field defined on $\left(\Theta^{\exp }, \mathcal{T}^{\exp }, \mathcal{P}^{\exp }\right)$, such that, for all $\mathbf{x}$ in $\Omega,\left[\AA^{\exp }(\mathbf{x})\right]=\left[A^{0}\right]+\left[\underline{\mathcal{L}}^{\exp }\right]^{T}\left[\mathcal{L}\left(\mathbf{x} ; \delta^{\exp }, L_{c}^{\exp }\right)\right]^{T}\left[G^{\text {pert }}\right]\left[\mathcal{L}\left(\mathbf{x} ; \delta^{\exp }, L_{c}^{\exp }\right)\right]\left[\underline{\mathcal{L}}^{\exp }\right]$. We have taken $\delta^{\exp }=0.25, L_{c}^{\exp }=0.33$, and $\left[G^{\text {pert }}\right]$ is the positive-definite symmetric $(6 \times 6)$ random matrix defined in [42] such that $E\left\{\left[G^{\text {pert }}\right]\right\}=\left[I_{6}\right]$ and whose statistical fluctuations are controlled by the dispersion parameter $\delta^{\text {pert }}$ which is chosen equal to 0.3 . It should be noted that the total statistical fluctuations of this random field is controlled by two multiplicative random matrices $\left[G\left(\mathbf{x} ; \delta^{\exp }, L_{c}^{\exp }\right)\right]=\left[\mathcal{L}\left(\mathbf{x} ; \delta^{\exp }, L_{c}^{\exp }\right)\right]^{T}\left[\mathcal{L}\left(\mathbf{x} ; \delta^{\exp }, L_{c}^{\exp }\right)\right]$ and $\left[G^{\text {pert }}\right]$, whose dispersion parameters are $\delta^{\exp }=0.25$ and $\delta^{\text {pert }}=0.3$. For the anisotropic material, the $(6 \times 6)$ real matrix $\left[\underline{A}^{\exp }\right]=\left(1-\varepsilon_{0}\right)\left[\underline{A}^{\exp }\right]$ of the mean model, such that $\left[\underline{A}^{\exp }\right]=\left[\underline{\mathcal{L}}^{\exp }\right]^{T}\left[\underline{\mathcal{L}}^{\exp }\right]$, is defined as

$$
\left[\underline{A}^{\exp }\right]=10^{10} \times\left[\begin{array}{cccccc}
3.3617 & 1.7027 & 1.3637 & -0.1049 & -0.2278 & 2.1013 \\
1.7027 & 1.6092 & 0.7262 & 0.0437 & -0.1197 & 0.8612 \\
1.3637 & 0.7262 & 1.4653 & -0.1174 & -0.1506 & 1.0587 \\
-0.1049 & 0.0437 & -0.1174 & 0.1319 & 0.0093 & -0.1574 \\
-0.2278 & -0.1197 & -0.1506 & 0.0093 & 0.1530 & -0.1303 \\
2.1013 & 0.8612 & 1.0587 & -0.1574 & -0.1303 & 1.7446
\end{array}\right] .
$$


The matrix defined by Eq. (43) corresponds to the mean value of the elasticity tensor of the anisotropic random microstructure presented in [44]. Finally, we consider $\nu_{\exp }=200$ experimental data sets $\mathbf{u}^{\exp , 1}, \ldots, \mathbf{u}^{\exp , \nu \exp }$ considered as independent realizations of the random vector $\mathbf{U}^{\exp }$ defined on probability space $\left(\Theta^{\exp }, \mathcal{T}^{\exp }, \mathcal{P}^{\exp }\right)$. Each experimental data set $\mathbf{u}^{\exp , \ell}$ is then generated as the realization $\mathbf{U}^{\exp }\left(\theta_{\ell}^{\exp }\right)$ of the observation vector in $\mathrm{R}^{m_{\mathrm{obs}}}$, that is to say, is such that $\mathbf{u}^{\exp , \ell}=\mathbf{h}^{\text {obs }}\left(\mathrm{V}^{\exp }\left(\theta_{\ell}^{\exp }\right)\right)$ in which $\theta_{1}^{\exp }, \ldots, \theta_{\nu_{\exp }}^{\exp }$ are $\nu_{\exp }$ independent realizations in $\Theta^{\exp }$ and where $\mathrm{V}^{\exp }$ is the random vector representing the finite family of random matrices $\left\{\left[\AA^{\exp }(\mathbf{x})\right], \mathbf{x} \in \mathcal{I}\right\}$.

\subsection{Identification with experimental data of an optimal PASM in the constructed family}

The optimization problem defined in Step 2 of Section 3 is constructed using the least-square method and allows the identification of the optimal PASM to be carried out using the experimental data. This optimization problem is solved by the trial method. The cost function $\mathbf{w} \mapsto J(\mathbf{w})$ (defined in [48]) is computed for $\delta \in\{0.24,0.27,0.30,0.33,0.36,0.39,0.42,0.45,0.48,0.51,0.55\}$, for $L_{c} \in$ $\{0.2,0.24,0.28,0.3,0.31,0.32,0.33,0.34,0.35,0.36,0.4\}$ and for $[\underline{A}]=\left[\underline{A}^{\exp }\right]$ (note that $[\underline{A}]$ is fixed and is not free in the optimization problem). For each trial point $\mathbf{w}$, the value of the cost function $J(\mathbf{w})$ is estimated using the stochastic numerical model $\mathbf{U}^{\text {obs,PASM }}(\mathbf{w})=\mathbf{h}^{\text {obs }}\left(\mathrm{V}^{\mathrm{PASM}}(\mathbf{w})\right)$ which is solved by the Monte Carlo method with 1000 independent realizations of random vector $\mathrm{V}^{\mathrm{PASM}}(\mathbf{w})$. The optimal value $\mathbf{w}^{\mathrm{opt}}=\left(\delta^{\mathrm{opt}}, L_{c}^{\mathrm{opt}}\right)$, corresponding to the minimum of the cost function, is obtained for $\delta^{\mathrm{opt}}=0.42$ and $L_{c}^{\mathrm{opt}}=0.34$. Fig. 2 displays the graph of the cost function $\left(\delta, L_{c}\right) \mapsto J\left(\delta, L_{c}\right)$.

\subsection{Construction of the statistical reduced-order optimal PASM}

We apply Step 3 of the methodology presented in Section 3. The optimal PASM $\mathrm{V}^{\mathrm{PASM}}\left(\mathbf{w}^{\mathrm{opt}}\right)$ is simply denoted by $\mathrm{V}^{\mathrm{PASM}}$. Its mean value $\underline{\mathrm{V}}=E\left\{\mathrm{~V}^{\mathrm{PASM}}\left(\mathbf{w}^{\mathrm{opt}}\right)\right\}$ is a vector in $\mathrm{R}^{36} 288$ and its covariance matrix $\left[C_{\mathrm{V}^{\mathrm{PASM}}}\right]$ is a $(36288 \times 36288)$ real symmetric matrix. These two second-order moments are estimated with the optimal PASM using $\nu_{\mathrm{KL}}=1000$ independent realizations. The dominant eigenspace of the eigenvalue problem $\left[C_{\mathrm{V}^{\mathrm{PASM}}}\right] \mathrm{W}^{j}=\lambda_{j} \mathrm{~W}^{j}$ is solved by using the usual subspace iteration method without assembling matrix $\left[C_{\mathrm{V}}\right.$ PASM $]$. The $(36288 \times n)$ real matrix $[\mathrm{W}]=\left[\mathrm{W}^{1} \ldots \mathrm{W}^{n}\right]$ of the $n$ eigenvectors associated with the $n$ largest eigenvalues $\lambda_{1} \geq \lambda_{2} \geq \ldots \geq \lambda_{n}>0$ is such that $[\mathrm{W}]^{T}[\mathrm{~W}]=\left[I_{n}\right]$. Fig. 3 displays the graph of the relative error function $n \mapsto \operatorname{err}(n)=1-\left(\sum_{j=1}^{n} \lambda_{j}\right) / \operatorname{tr}\left[C_{\mathrm{V}^{\mathrm{PASM}}}\right]$ related to the 


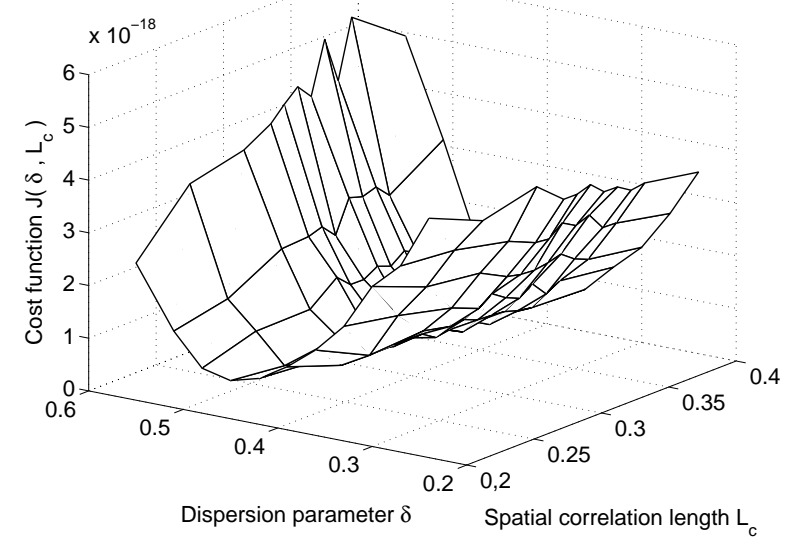

Figure 2: Cost function $\left(\delta, L_{c}\right) \mapsto J\left(\delta, L_{c}\right)$ for the identification of the optimal PASM using the experimental data sets.

convergence (with respect to $n$ ) of the expansion of random vector $\mathrm{V}^{\mathrm{PASM}}$ corresponding to the optimal PASM. This figure shows that a reasonable convergence is reached for $n=550$.

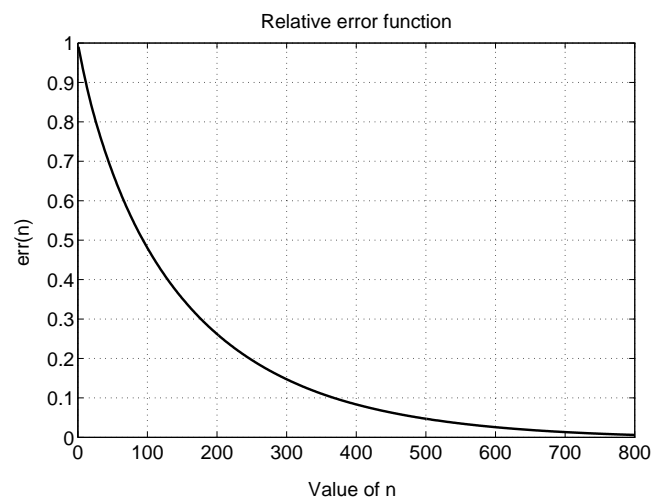

Figure 3: Graph of the error function $n \mapsto \operatorname{err}(n)$.

8.7. Construction of the PCE with deterministic VVC of the reduced-order optimal PASM

We apply Step 4 of the methodology presented in Section 3. The calculations of (1) the value $N_{g}$ of the length of germ $\Xi=\left(\Xi_{1}, \ldots, \Xi_{N_{g}}\right)$, (2) the max- 
imum degree $N_{d}$ of the polynomial chaos $\Psi_{\alpha}(\boldsymbol{\Xi})$, and (3) the optimal values $\underline{\mathbf{y}}^{1}, \ldots, \underline{\mathbf{y}}^{N}$ of the deterministic VVC in $\mathrm{R}^{n}$ (with $n=550$ ) of the PCE $\boldsymbol{\eta}^{\text {chaos }}(N)=$ $\sum_{\alpha=1}^{N} \mathbf{y}^{\bar{\alpha}} \Psi_{\alpha}(\boldsymbol{\Xi})$, are performed as explained in Step 4 of Section 3 and in [48], with $\nu_{\mathrm{KL}}=1000$ and $\nu=11000$. The convergence is obtained for $N_{g}=4$ (as in [48]). Fig. 4 displays the graph of the $L^{1}$-error function $N_{d} \mapsto \operatorname{err}\left(N_{g}, N_{d}\right)$ for random vector $\boldsymbol{\eta}^{\text {chaos }}(N)$ with $N=h\left(N_{g}, N_{d}\right)$ and for $N_{g}=4$. It can be seen that convergence is obtained for $N_{d}=20$. At convergence $\left(N_{g}=4\right.$ and $\left.N_{d}=20\right)$, there are $N=10625$ deterministic VVC in the PCE of $\boldsymbol{\eta}^{\text {chaos }}(N)$, that is to say $5843750=10625 \times 550$ real coefficients which have been identified. It should be noted that there are $N_{g}=4$ independent Gaussian germs, inducing $N=10625$ uncorrelated non-Gaussian (but dependent) random variables used in the PCE, value $\left(N_{g}=4\right)$ which has to be compared to the 36288 independent Gaussian germs used to generate $\mathrm{V}^{\mathrm{PASM}}$ (the optimal non-Gaussian PASM). Fig. 5 is related to the convergence analysis with respect to the number $N=h\left(N_{g}, N_{d}\right)$ of chaos. Each figure shows the comparison of the graph of the optimal PASM pdf $e \mapsto$ $\log _{10}\left(p_{\eta_{j}^{\text {PASM }}}(e)\right)$ with the graph of the pdf $e \mapsto \log _{10}\left(p_{\eta_{j}^{\text {chaos }}(N)}\left(e ; \underline{\mathbf{y}}^{1}, \ldots, \underline{\mathbf{y}}^{N}\right)\right)$ estimated using the PCE with $N=h\left(N_{g}, N_{d}\right)$ chaos. The figures show the comparisons for the coordinates $j=1$ and 550 , for $N_{g}=4$ and for $N_{d}=9(N=714)$, $N_{d}=20(N=10625)$ and for $N_{d}=22(N=14949)$. It can be seen again a good convergence of the probability density function for these two coordinates obtained for $N_{g}=4$ and $N_{d}=20$ corresponding to the value $N=10625$. The quality of the convergence is similar for the other 548 coordinates.

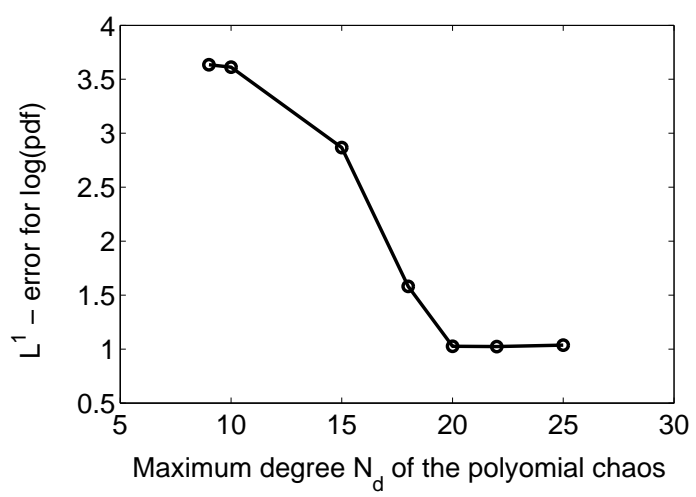

Figure 4: $L^{1}$-error function $N_{d} \mapsto \operatorname{err}\left(N_{g}, N_{d}\right)$ for random vector $\eta^{\text {chaos }}(N)$ with $N=$ $h\left(N_{g}, N_{d}\right)$ and for $N_{g}=4$. 

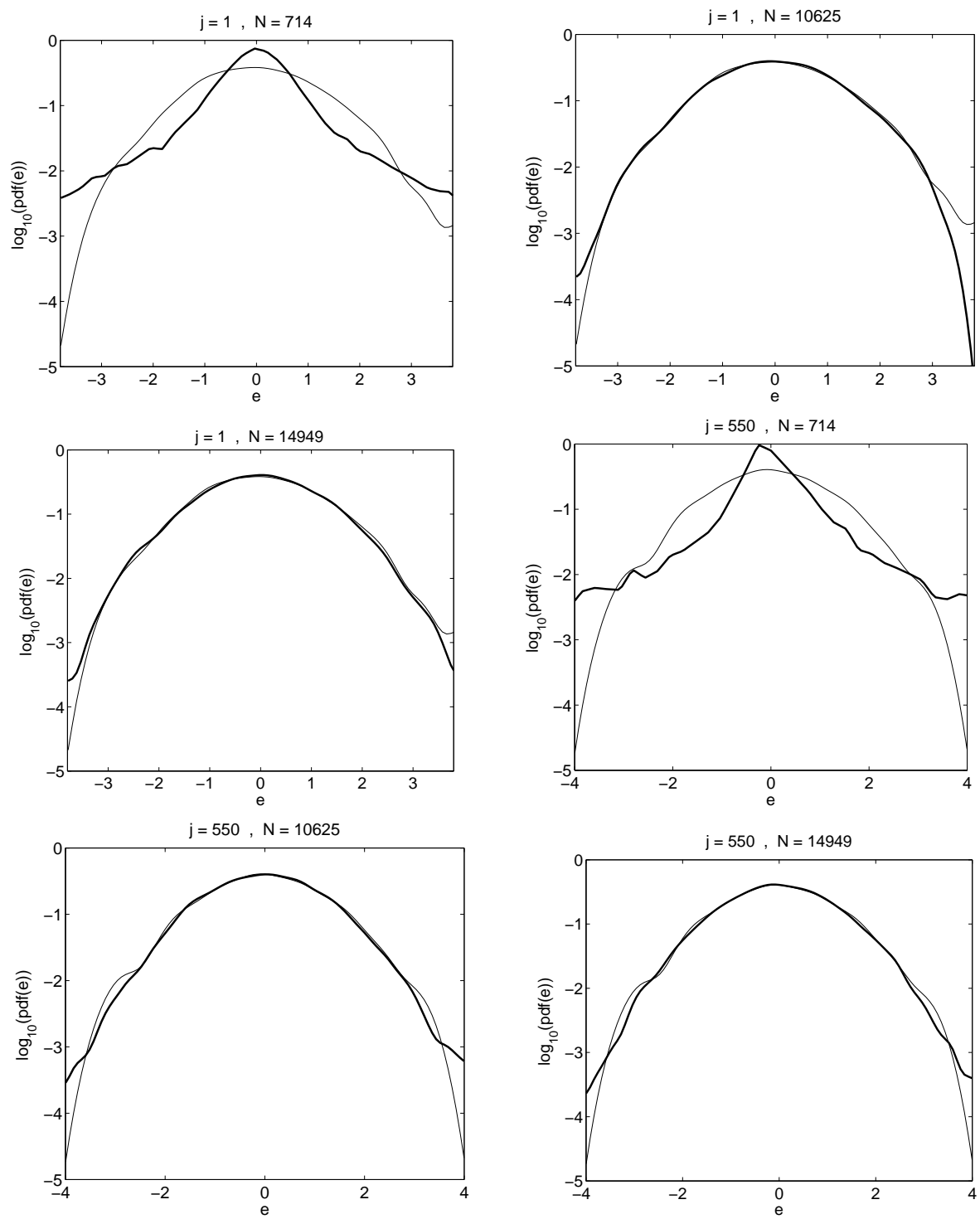

Figure 5: Convergence analysis with respect to the number $N$ of chaos. Comparisons of the graph of the optimal PASM $p d f e \mapsto p_{\eta_{j}^{\text {PASM }}}(e)$ (thin solid line) with the graph of the pdf $e \mapsto p_{\eta_{j}^{\text {chaos }}}(e)$ estimated using the PCE with $N$ chaos (thick solid line), for different values of $N$ and for given coordinate $j$ (the values of $j$ and $N$ are indicated at the top of each figure). Vertical axis: $\log _{10}$ of the pdf. Horizontal axis: value e of $\eta_{j}$. 


\subsection{Prior probability model of the random VVC}

Section 5 is used with $n=550, N=10625, \varepsilon=1.2, \nu^{\prime}=10000$ and $\nu=3000$. As explained in Section 5.4, each matrix of the family $\left\{\left[\mathbf{A}^{\text {prior }}\left(\mathbf{x}^{1} ; \theta_{\ell^{\prime}}^{\prime}, \theta_{\ell}\right)\right], \ldots\right.$, $\left.\left[\mathbf{A}^{\text {prior }}\left(\mathbf{x}^{N_{p}} ; \theta_{\ell^{\prime}}^{\prime}, \theta_{\ell}\right)\right]\right\}$ must be positive definite (property $\left.\mathcal{P}_{\text {rop }}\right)$. For fixed $\theta_{\ell^{\prime}}^{\prime}$, the subset $\left\{\theta_{\ell_{1}}, \ldots, \theta_{\ell_{\widetilde{\nu}\left(\ell^{\prime}\right)}}\right\}$ of $\left\{\theta_{1}, \ldots, \theta_{\nu}\right\}$ has been determined with the stochastic computational model in order that property $\mathcal{P}_{\text {rop }}$ be verified. Fig. 6 displays the graph of function $\ell^{\prime} \mapsto \widetilde{\nu}\left(\ell^{\prime}\right)$ from $\left\{1, \ldots, \nu^{\prime}\right\}$ into $\{1, \ldots, \nu\}$. It can be seen that the average value is about 340, which compared to $\nu=3000$, corresponds to a significant rate of rejection. Such a rate can easily be understood taking

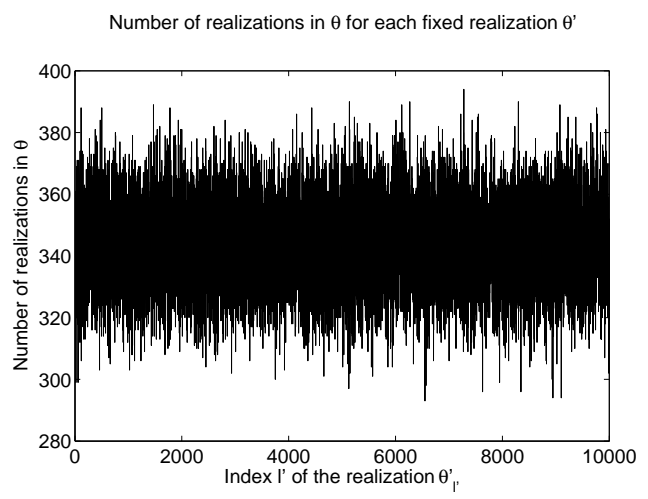

Figure 6: Graph of function $\ell^{\prime} \mapsto \widetilde{\nu}\left(\ell^{\prime}\right)$ related to property $\mathcal{P}_{\text {rop }}$.

into account the high value of $\varepsilon$ which has been fixed to 1.2. The total number of realizations in $\Theta^{\prime} \times \Theta$ is $\sum_{\ell^{\prime}=1}^{\nu^{\prime}} \widetilde{\nu}\left(\ell^{\prime}\right)=3439684$. We compare the experimental data $U_{k}^{\exp }$ with the random responses $U_{k}^{\text {PASM }}$ and $U_{k}^{\text {prior }}$ associated with $\mathrm{V}^{\mathrm{PASM}}$ (the optimal PASM represented by the optimal chaos expansion) and $\mathrm{V}^{\text {prior }}$ (the prior model), respectively. In order to limit the number of figures presented in the paper, we have selected the observed degrees of freedom $k$ which correspond to the $x_{2}$-displacement of nodes 9, 17, 25 and 37 (among the 25 nodes located inside the face of equation $\left.x_{1}=0\right)$. An estimation $u_{k}^{\text {obs }} \mapsto \widehat{p}_{U_{k}^{\exp }}\left(u_{k}^{\mathrm{obs}}\right)$ of the probability density function of the random variable $U_{k}^{\exp }$ is carried out using experimental data $u_{k}^{\exp , 1}, \ldots, u_{k}^{\exp , \nu_{\exp }}$ (see Section 2-(3)) and the kernel estimation method [6]. The probability density function $u_{k}^{\mathrm{obs}} \mapsto p_{U_{k}^{\mathrm{obs}, \mathrm{PASM}}}\left(u_{k}^{\mathrm{obs}}\right)$ is estimated as explained in Section 3-Step 4-(iii). The probability density function $u_{k}^{\mathrm{obs}} \mapsto p_{U_{k}^{\mathrm{obs}} \text {,prior }}\left(u_{k}^{\mathrm{obs}}\right)$ is estimated as explained in Section 5.5. Fig. 7 shows the comparison of $u_{k}^{\text {obs }} \mapsto \widehat{p}_{U_{k}^{\exp }}\left(u_{k}^{\mathrm{obs}}\right)$ with $u_{k}^{\mathrm{obs}} \mapsto p_{U_{k}^{\mathrm{obs}, \mathrm{PASM}}}\left(u_{k}^{\mathrm{obs}}\right)$ and 

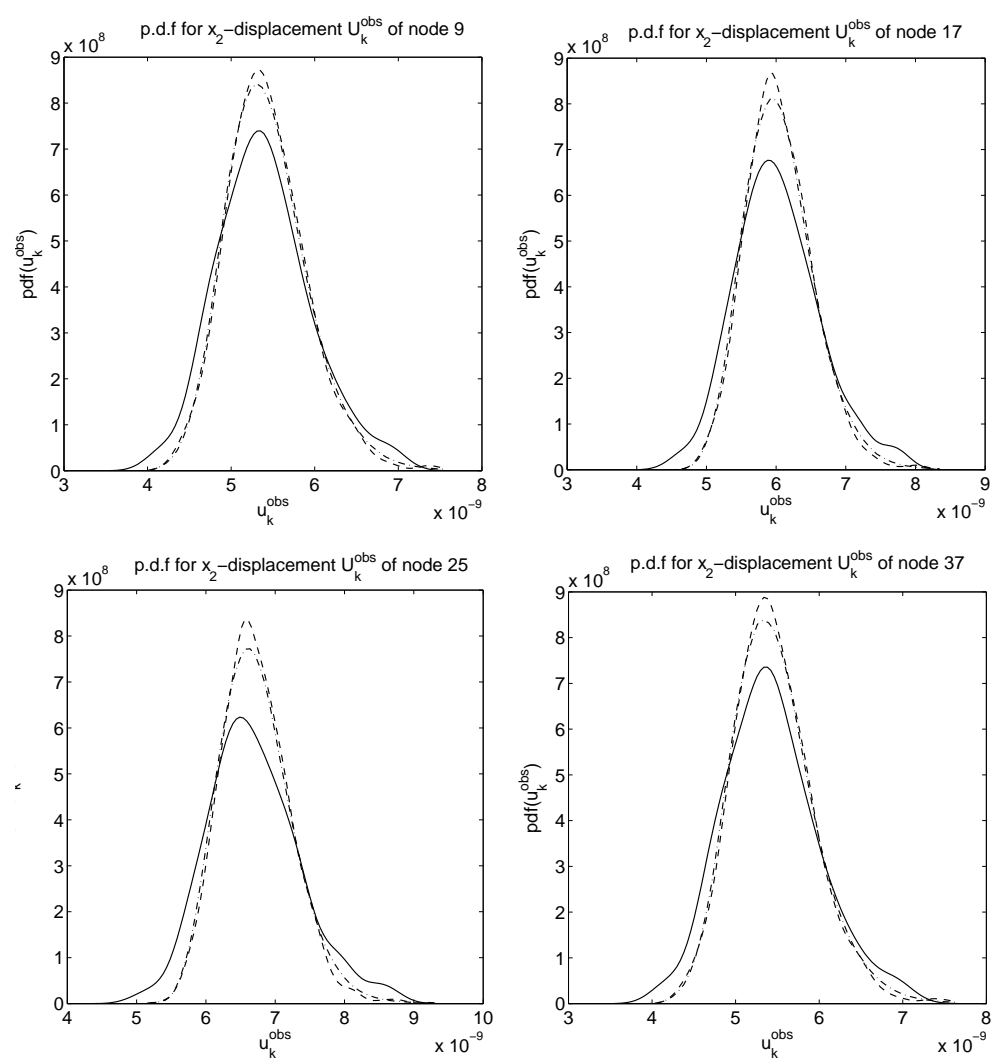

Figure 7: For observed degrees of freedom $k$ corresponding to the $x_{2}$-displacement of nodes 9 , 17,25 and 37 , graphs of $u_{k}^{\text {obs }} \mapsto \widehat{p}_{U_{k}^{\text {exp }}}\left(u_{k}^{\text {obs }}\right)$ (thin solid lines), $u_{k}^{\text {obs }} \mapsto p_{U_{k}^{\text {obs }} \text { PASM }}\left(u_{k}^{\text {obs }}\right)$ (dashed lines), $u_{k}^{\mathrm{obs}} \mapsto p_{U_{k}^{\mathrm{obs}} \text {, prior }}\left(u_{k}^{\mathrm{obs}}\right)($ dashed dotted lines).

$u_{k}^{\text {obs }} \mapsto p_{U_{k}^{\text {obs,prior }}}\left(u_{k}^{\text {obs }}\right)$. As explained in Section 8.4, these four figures show that there are significant differences between the experiments and the PASM for the observed degrees of freedom. It can also be seen that the prior model is not yet sufficient to correctly fit the experiments.

\subsection{Posterior probability model of the random VVC using the classical Bayesian approach}

We compare the experimental data $U_{k}^{\text {exp }}$ with the random responses $U_{k}^{\text {prior }}$ and $U_{k}^{\text {post }}$ associated with the prior model $\mathrm{V}^{\text {prior }}$ and the posterior model $\mathrm{V}^{\text {post }}$ computed with the method presented in Section 6. For the observed degrees of freedom $k$, we use the estimations $u_{k}^{\mathrm{obs}} \mapsto \widehat{p}_{U_{k}^{\exp }}\left(u_{k}^{\mathrm{obs}}\right)$ and $u_{k}^{\mathrm{obs}} \mapsto p_{U_{k}^{\mathrm{obs}} \text { prior }}\left(u_{k}^{\mathrm{obs}}\right)$ calculated in Sec- 

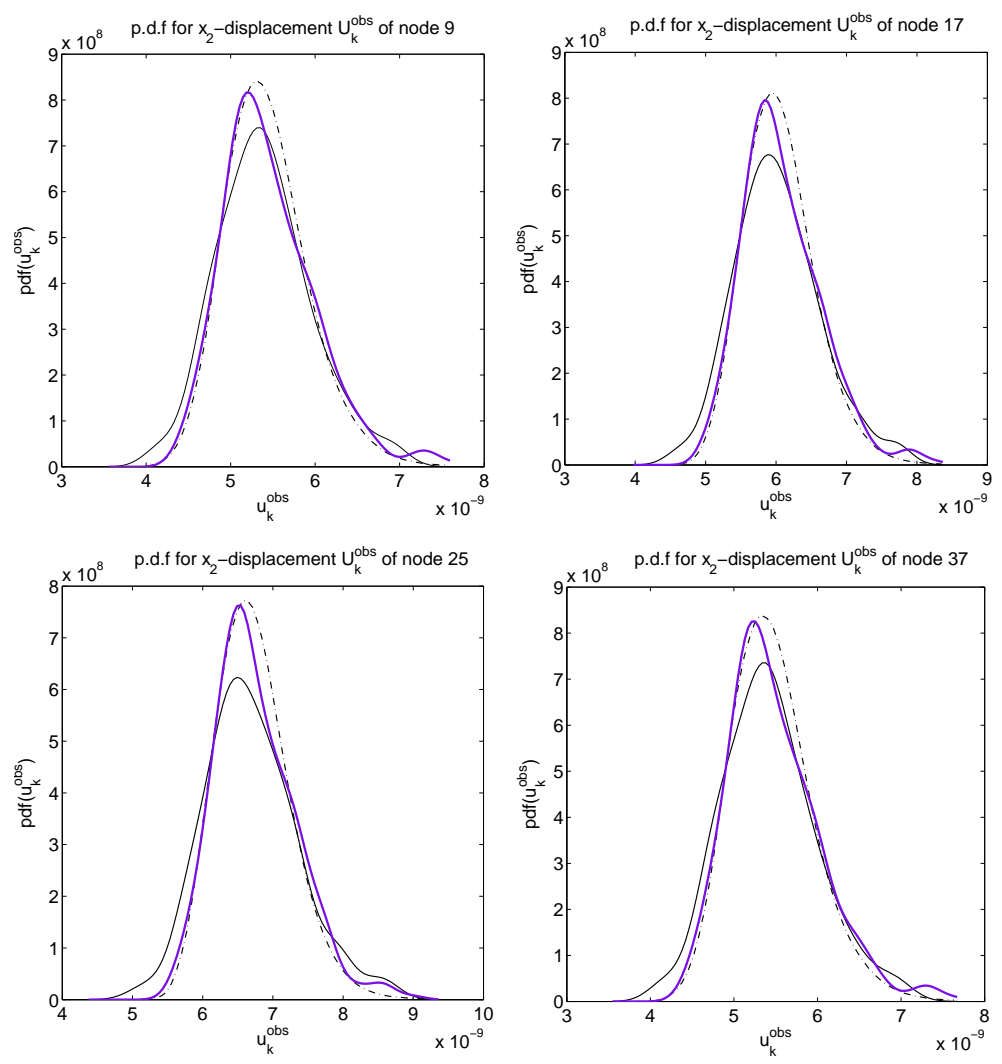

Figure 8: For observed degrees of freedom $k$ corresponding to the $x_{2}$-displacement of nodes 9,17 , 25 and 37 , graphs of $u_{k}^{\text {obs }} \mapsto \widehat{p}_{U_{k}^{\text {exp }}}\left(u_{k}^{\text {obs }}\right)$ (thin solid lines), $u_{k}^{\text {obs }} \mapsto p_{U_{k}^{\text {obs, prior }}}\left(u_{k}^{\text {obs }}\right)$ (dashed dotted lines), $u_{k}^{\mathrm{obs}} \mapsto p_{U_{k}^{\mathrm{obs}} \text { post }}\left(u_{k}^{\mathrm{obs}}\right)$ computed with Section 6.4 (blue thick solid lines).

tion 8.8. The probability density function $u_{k}^{\text {obs }} \mapsto p_{U_{k}^{\text {obs,post }}}\left(u_{k}^{\text {obs }}\right)$ is estimated as explained in Section 6.4. Fig. 8 shows the comparison of $u_{k}^{\text {obs }} \mapsto \widehat{p}_{U_{k}^{\exp }}\left(u_{k}^{\text {obs }}\right)$ with $u_{k}^{\mathrm{obs}} \mapsto p_{U_{k}^{\mathrm{obs}} \text {,prior }}\left(u_{k}^{\mathrm{obs}}\right)$ and $u_{k}^{\mathrm{obs}} \mapsto p_{U_{k}^{\mathrm{obs}} \text { post }}\left(u_{k}^{\mathrm{obs}}\right)$. These figures show that the posterior model improves the prior model a bit, but is not sufficiently good taken into account that the comparisons are relative to observed degrees of freedom for which experimental data are used in the identification procedure. 

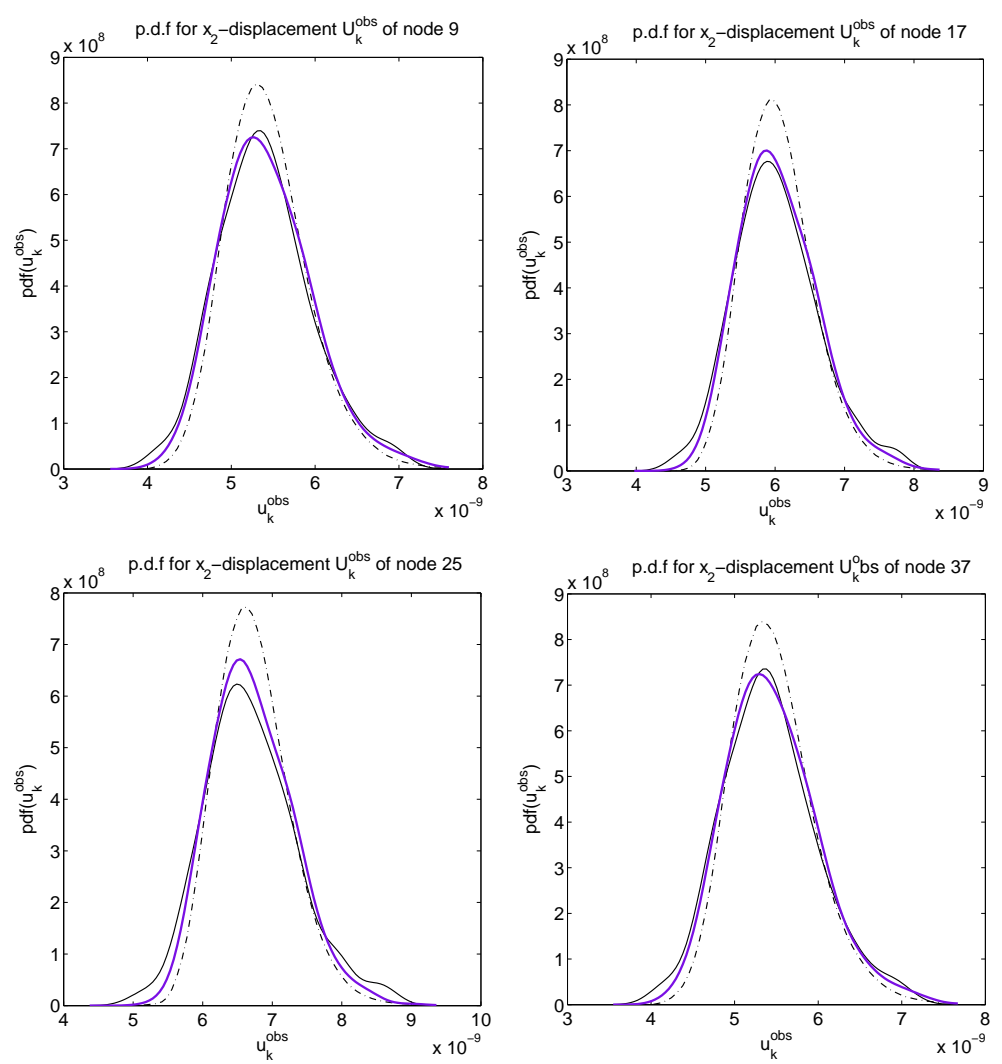

Figure 9: For observed degrees of freedom $k$ corresponding to the $x_{2}$-displacement of nodes 9,17 , 25 and 37 , graphs of $u_{k}^{\text {obs }} \mapsto \widehat{p}_{U_{k}}^{\exp }\left(u_{k}^{\text {obs }}\right)$ (thin solid lines), $u_{k}^{\text {obs }} \mapsto p_{U_{k}^{\text {obs,prior }}}\left(u_{k}^{\text {obs }}\right)$ (dashed dotted lines), $u_{k}^{\mathrm{obs}} \mapsto p_{U_{k}^{\mathrm{obs}} \text {,post }}\left(u_{k}^{\mathrm{obs}}\right)$ computed with Section 7 (blue thick solid lines).

8.10. Posterior probability model of the random VVC using a new approach derived from the Bayesian approach

We compare the experimental data $U_{k}^{\text {exp }}$ with the random responses $U_{k}^{\text {prior }}$ and $U_{k}^{\text {post }}$ associated with the prior model $\mathrm{V}^{\text {prior }}$ and the posterior model $\mathrm{V}^{\text {post }}$ computed with the method presented in Section 7. For the observed degrees of freedom $k$,we use the estimations $u_{k}^{\text {obs }} \mapsto \widehat{p}_{U_{k}^{\exp }}\left(u_{k}^{\text {obs }}\right)$ and $u_{k}^{\text {obs }} \mapsto p_{U_{k}^{\text {obs,prior }}}\left(u_{k}^{\text {obs }}\right)$ calculated in Section 8.8. The probability density function $u_{k}^{\mathrm{obs}} \mapsto p_{U_{k}^{\mathrm{obs} p \text { post }}}\left(u_{k}^{\mathrm{obs}}\right)$ is estimated as explained in Section 7. Fig. 9 shows the comparison of $u_{k}^{\text {obs }} \mapsto \widehat{p}_{U_{k}^{\exp }}\left(u_{k}^{\text {obs }}\right)$ with $u_{k}^{\text {obs }} \mapsto p_{U_{k}^{\text {obs,prior }}}\left(u_{k}^{\text {obs }}\right)$ and $u_{k}^{\text {obs }} \mapsto p_{U_{k}^{\text {obs,post }}}\left(u_{k}^{\text {obs }}\right)$. These figures show that the posterior model significantly improves the prior model and that the comparisons 
with the experiments are good.

\subsection{Quality assessment of the two posterior stochastic models}

In Sections 8.9 and 8.10, we have compared the probability density functions of observations, calculated with the two proposed posterior models. For these two models, the observations (for which experimental data are available) are used to identify the models. In order to give a quality assessment of the posterior stochastic models, we present comparisons for degrees of freedom which are not observed (that is to say which are not used in the identification procedure of the posterior stochastic models). We then consider degrees of freedom $k$, such that $m_{\mathrm{obs}}+1 \leq k \leq m$, corresponding to the $x_{2}$-displacement of the nodes $72,74,170$ and 174 for which the coordinates are $(0.1667,0.500,0.1667)$, $(0.1667,0.500,0.500),(0.500,0.500,0.1667)$ and $(0.500,0.500,0.8333)$. These four nodes are located inside the cube. Figs. 10 and 11 show the comparisons of $u_{k}^{\text {nobs }} \mapsto \widehat{p}_{U_{k}^{\exp }}\left(u_{k}^{\text {nobs }}\right)$ with $u_{k}^{\text {nobs }} \mapsto p_{U_{k}^{\text {nobs, prior }}}\left(u_{k}^{\text {nobs }}\right)$ and $u_{k}^{\text {nobs }} \mapsto p_{U_{k}^{\text {nobs }, \text { post }}}\left(u_{k}^{\text {nobs }}\right)$ computed with Sections 8.9 and 8.10. The four figures in Fig. 10 show that the quality of the posterior stochastic model, identified with the Bayesian approach for degrees of freedom which have not be used to identify it, is not really good while the four figures in Fig. 11 show that the quality is good enough for the posterior stochastic model identified with the proposed new approach derived from the Bayesian method.

\section{Conclusions}

A methodology has been proposed for the identification of a Bayesian posterior of a high-dimension PCE of a non-Gaussian tensor-valued random field using partial and limited experimental data, through a stochastic boundary value problem. We have validated the methodology of this very challenging problem for which the joint probability distribution of several millions of dependent non-gaussian random variables has to be identified. The first four steps of the methodology have been introduced and validated in a previous paper. In the present work, we have developed two approaches for the last step consisting in the identification of the Bayesian posteriors from a prior model, and which constitute novel results due to the high-dimension aspects. In addition, we have proposed and validated a new approach derived from the Bayesian method which is very efficient in high dimension. 

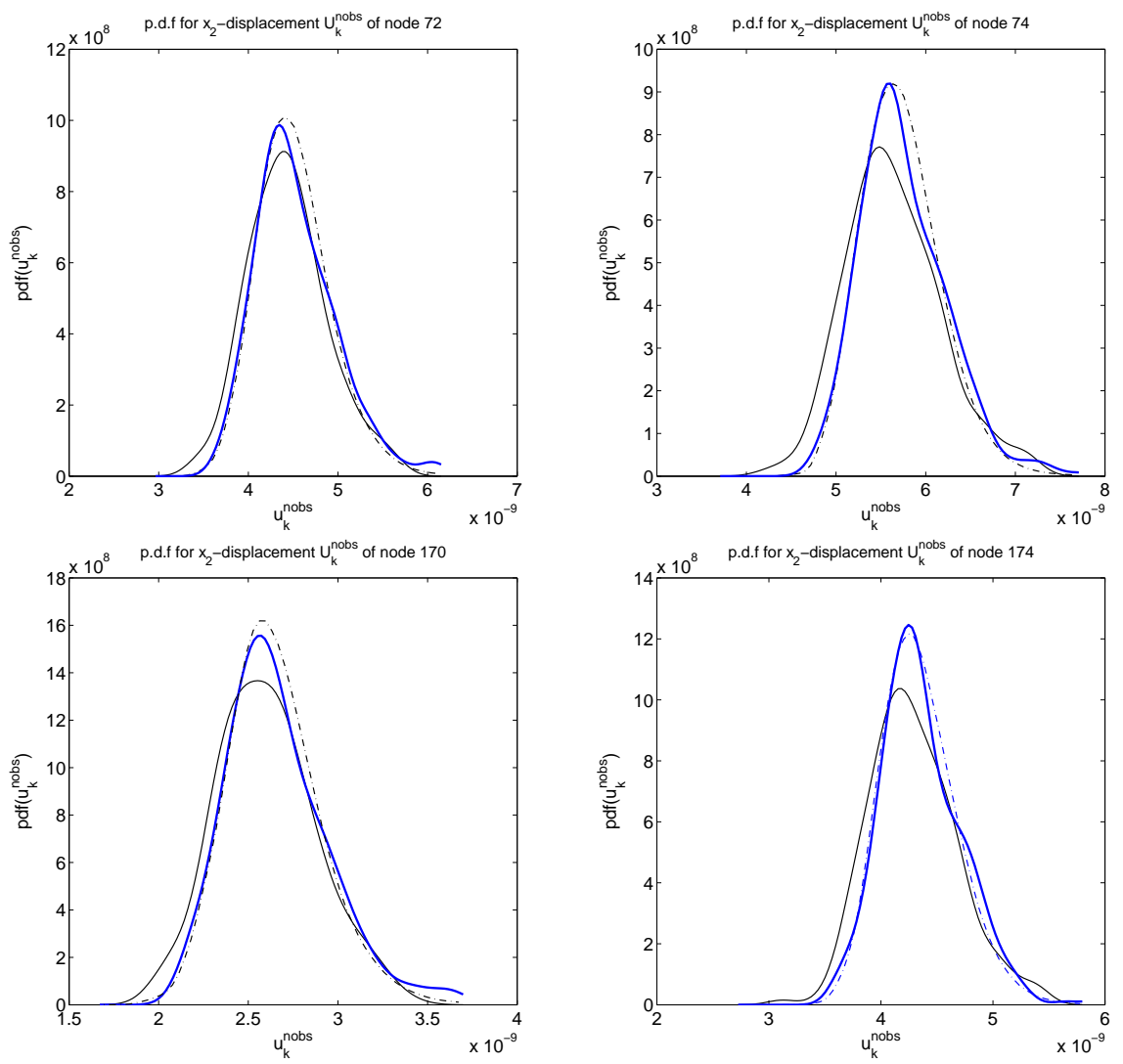

Figure 10: For degrees of freedom $k$ not used in the identification of the posterior stochastic model and corresponding to the $x_{2}$-displacement of nodes $72,74,170$ and 174, graphs of $u_{k}^{\text {nobs }} \mapsto \widehat{p}_{U_{k}^{\exp }}\left(u_{k}^{\text {nobs }}\right)$ (thin solid lines), $u_{k}^{\text {nobs }} \mapsto p_{U_{k}^{\text {nobs,prior }}}\left(u_{k}^{\text {nobs }}\right)$ (dashed dotted lines), $u_{k}^{\text {nobs }} \mapsto p_{U_{k}^{\text {nobs,post }}}\left(u_{k}^{\text {nobs }}\right)$ computed in Section 8.9 (blue thick solid lines).

\section{Acknowledgement}

This research was supported by the "Agence Nationale de la Recherche", Contract TYCHE, ANR-2010-BLAN-0904.

\section{A. Appendix: Estimation of a probability density function on $R^{m}$ using the multivariate Gaussian kernel density estimation}

In this appendix, we summarize the nonparametric estimation of a multivariate probability density function using the multivariate Gaussian kernel density estimation (see for instance $[6,52])$. Such a method is used to estimate $p_{\mathbf{U}^{\mathrm{obs}} \mid \mathrm{Y}}\left(\left.\mathbf{u}^{\exp , \ell}\right|_{\mathrm{Y}}\right)$ 

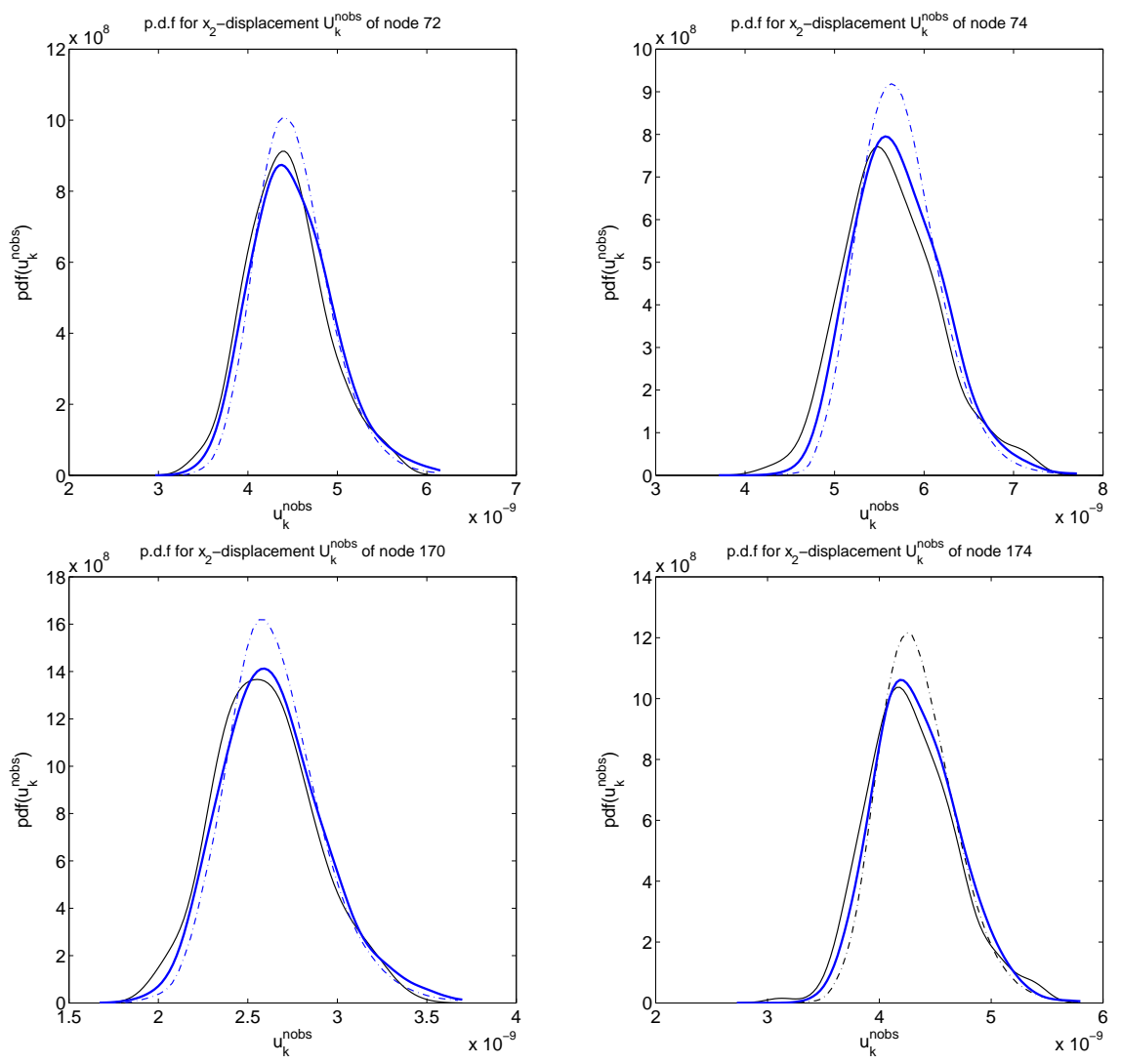

Figure 11: For degrees of freedom $k$ not used in the identification of the posterior stochastic model and corresponding to the $x_{2}$-displacement of nodes 72, 74, 170 and 174, graphs of $u_{k}^{\text {nobs }} \mapsto \widehat{p}_{U_{k}^{\exp }}\left(u_{k}^{\text {nobs }}\right)$ (thin solid lines), $u_{k}^{\text {nobs }} \mapsto p_{U_{k}^{\text {nobs,prior }}}\left(u_{k}^{\text {nobs }}\right)$ (dashed dotted lines), $u_{k}^{\text {nobs }} \mapsto p_{U_{k}^{\text {nobs,post }}}\left(u_{k}^{\text {nobs }}\right)$ computed in Section 8.10 (blue thick solid lines).

(see Eq. (29)) for given $\mathbf{u}^{\text {exp }, \ell}$ and for given $y$.

Let $\mathbf{S}=\left(S_{1}, \ldots, S_{m}\right)$, with $m>1$, be any second-order random variable defined on $(\Theta, \mathcal{T}, \mathcal{P})$ with values in $\mathrm{R}^{m}$ (the components are statistically dependent and $\mathbf{S}$ is not a Gaussian random vector). Let $\mathbf{S}\left(\theta_{1}\right), \ldots, \mathbf{S}\left(\theta_{\nu}\right)$ be $\nu$ independent realizations of $\mathbf{S}$ with $\theta_{1}, \ldots, \theta_{\nu}$ in $\Theta$. Let $P_{\mathbf{S}}(d \mathbf{s})=p_{\mathbf{S}}(\mathbf{s}) d \mathbf{s}$ be the probability distribution defined by an unknown probability density function $\mathbf{S} \mapsto p_{\mathbf{S}}(\mathbf{s})$ on $\mathrm{R}^{m}$, with respect to the Lebesgue measure $d \mathbf{s}$ on $\mathrm{R}^{m}$. For $\mathbf{s}^{0}$ fixed in $\mathrm{R}^{m}$ and for $\nu$ sufficiently large, the multivariate kernel density estimation allows the nonparametric estimation $\widehat{p}_{\mathbf{S}}\left(\mathbf{s}^{0}\right)$ of $p_{\mathbf{S}}\left(\mathbf{s}^{0}\right)$ to be carried out using the $\nu$ independent realizations 
$\mathbf{S}\left(\theta_{1}\right), \ldots, \mathbf{S}\left(\theta_{\nu}\right)$. The first step consists in performing a rotation and a normalization of data in the principal component axes. The second step is devoted to the Gaussian kernel density for each direction.

Let $\underline{\widehat{\mathbf{S}}}$ and $\left[\widehat{C}_{\mathbf{S}}\right]$ be the usual statistical estimations of the mean value and the covariance matrix of the random vector $\mathbf{S}$ using the $\nu$ realizations. For instance, $\underline{\widehat{\mathbf{S}}}=\nu^{-1} \sum_{\ell=1}^{\nu} \mathbf{S}\left(\theta_{\ell}\right)$. It is assumed that $\left[\widehat{C}_{\mathbf{S}}\right]$ is a positive-definite symmetric $(m \times m)$ real matrix. Consequently, there is an orthogonal $(m \times m)$ real matrix $[\Phi]$ (that is to say $[\Phi][\Phi]^{T}=[\Phi]^{T}[\Phi]=\left[I_{m}\right]$ ) such that $\left[\widehat{C}_{\mathbf{S}}\right][\Phi]=[\Phi][\lambda]$ in which $[\lambda]$ is the diagonal matrix of the positive eigenvalues. Let $\mathbf{Q}=\left(Q_{1}, \ldots, Q_{m}\right)$ be the random vector such that

$$
\mathbf{S}=\underline{\widehat{\mathbf{S}}}+[\Phi] \mathbf{Q} \quad, \quad \mathbf{Q}=[\Phi]^{T}(\mathbf{S}-\underline{\widehat{\mathbf{S}}}) .
$$

We have $p_{\mathbf{S}}(\mathbf{s}) d \mathbf{s}=p_{\mathbf{Q}}(\mathbf{q}) d \mathbf{q}$ and since $|\operatorname{det}[\Phi]|=1$, we have $d \mathbf{s}=d \mathbf{q}$. Consequently, if we introduce $\mathbf{q}^{0}=[\Phi]^{T}\left(\mathbf{s}^{0}-\widehat{\widehat{\mathbf{S}}}\right)$, then $p_{\mathbf{S}}\left(\mathbf{s}^{0}\right)=p_{\mathbf{Q}}\left(\mathbf{q}^{0}\right)$ and therefore, the nonparametric estimation $\widehat{p}_{\mathbf{S}}\left(\mathbf{s}^{0}\right)$ of $p_{\mathbf{S}}\left(\mathbf{s}^{0}\right)$ is equal to the nonparametric estimation $\widehat{p}_{\mathbf{Q}}\left(\mathbf{q}^{0}\right)$ of $p_{\mathbf{Q}}\left(\mathbf{q}^{0}\right)$, that is to say,

$$
\widehat{p}_{\mathbf{S}}\left(\mathbf{s}^{0}\right)=\widehat{p}_{\mathbf{Q}}\left(\mathbf{q}^{0}\right)
$$

Using Eq. (A-1), the realizations $\mathbf{S}\left(\theta_{1}\right), \ldots, \mathbf{S}\left(\theta_{\nu}\right)$ are transformed in the realizations $\mathbf{Q}\left(\theta_{1}\right), \ldots, \mathbf{Q}\left(\theta_{\nu}\right)$ of random vector $\mathbf{Q}$ such that, for all $\ell$ in $\{1, \ldots, \nu\}$ $\mathbf{Q}\left(\theta_{\ell}\right)=[\Phi]^{T}\left(\mathbf{S}\left(\theta_{\ell}\right)-\underline{\widehat{\mathbf{S}}}\right)$. Eq. (A-2) shows that the initial problem is equivalent to the construction of the nonparametric estimation $\widehat{p}_{\mathbf{Q}}\left(\mathbf{q}^{0}\right)$ of $p_{\mathbf{Q}}\left(\mathbf{q}^{0}\right)$ using the realizations $\mathbf{Q}\left(\theta_{1}\right), \ldots, \mathbf{Q}\left(\theta_{\nu}\right)$ of random vector $\mathbf{Q}$. Let $\widehat{\mathbf{Q}}$ and $\left[\widehat{C}_{\mathbf{Q}}\right]$ be the usual statistical estimations of the mean value and the covariance matrix of the random vector $\mathbf{Q}$ using the $\nu$ independent realizations $\mathbf{Q}\left(\theta_{1}\right), \ldots, \mathbf{Q}\left(\theta_{\nu}\right)$. It can be seen that

$$
\widehat{\widehat{\mathbf{Q}}}=0 \quad, \quad\left[\widehat{C}_{\mathbf{Q}}\right]=[\lambda] .
$$

The second step consists in calculating $\widehat{p}_{\mathbf{Q}}\left(\mathbf{q}^{0}\right)$ using the the multivariate kernel density estimation which is written as

$$
\widehat{p}_{\mathbf{Q}}\left(\mathbf{q}^{0}\right)=\frac{1}{\nu} \sum_{\ell=1}^{\nu} \Pi_{k=1}^{m}\left\{\frac{1}{h_{k}} K\left(\frac{Q_{k}\left(\theta_{\ell}\right)-q_{k}^{0}}{h_{k}}\right)\right\},
$$

in which $h_{1}, \ldots, h_{m}$ are the smoothing parameters, $\mathbf{q}^{0}=\left(q_{1}^{0}, \ldots, q_{m}^{0}\right)$ and where $K$ is the kernel. For the multivariate Gaussian kernel density estimation, we have

$$
h_{k}=\sqrt{[\lambda]_{k k}}\left\{\frac{4}{\nu(2+m)}\right\}^{\frac{1}{4+m}} \quad, \quad K(v)=\frac{1}{\sqrt{2 \pi}} e^{-\frac{v^{2}}{2}} .
$$




\section{References}

[1] M. Arnst, R. Ghanem, C. Soize, Identification of Bayesian posteriors for coefficients of chaos expansion, Journal of Computational Physics, 229(9) (2010) 3134-3154.

[2] I. Babuska, R. Tempone, G.E. Zouraris, Solving elliptic boundary value problems with uncertain coefficients by the finite element method: the stochastic formulation, Computer Methods in Applied Mechanics and Engineering 194(12-16) (2005) 1251-1294.

[3] I. Babuska, F. Nobile, R. Tempone, A stochastic collocation method for elliptic partial differential equations with random input data, SIAM Journal on Numerical Analysis 45(3) (2007) 1005-1034.

[4] J.L. Beck, L.S. Katafygiotis, Updating models and their uncertainties. I: Bayesian statistical framework, Journal of Engineering Mechanics, 124(4) (1998) 455-461.

[5] J. M. Bernardo, A. F. M. Smith, Bayesian Theory, John Wiley \& Sons, Chichester, 2000.

[6] A.W. Bowman, A. Azzalini, Applied Smoothing Techniques for Data Analysis, Oxford University Press, 1997.

[7] B.P. Carlin, T.A. Louis, Bayesian Methods for Data Analysis (Third Edition), Chapman \& Hall / CRC Press, Boca Raton, 2009.

[8] C. Chen, D. Duhamel,C. Soize, Probabilistic approach for model and data uncertainties and its experimental identification in structural dynamics: Case of composite sandwich panels, Journal of Sound and Vibration, 294(1-2) (2006) 64-81.

[9] P. Congdon, Bayesian Statistical Modelling, Second Edition, John Wiley \& Sons, Chichester, 2007.

[10] S. Das, R. Ghanem, J. Spall, Asymptotic sampling distribution for polynomial chaos representation of data: A maximum-entropy and fisher information approach, SIAM Journal on Scientific Computing, 30(5) (2008) 2207-2234. 
[11] S. Das, R. Ghanem, S. Finette, Polynomial chaos representation of spatiotemporal random field from experimental measurements, Journal of Computational Physics, 228 (2009) 8726-8751.

[12] B.J. Debusschere, H.N. Najm, P.P. Pebay, et al., Numerical challenges in the use of polynomial chaos representations for stochastic processes, SIAM Journal on Scientific Computing, 26(2) (2004) 698-719.

[13] V. De Oliveira, B. Kedem, D.A. Short, Bayesian prediction of transformed gaussian random fields, Journal of the American Statistical Association, 92(440) (1997) 1422-1433.

[14] C. Desceliers, R. Ghanem, C. Soize, Maximum likelihood estimation of stochastic chaos representations from experimental data, International Journal for Numerical Methods in Engineering, 66(6) (2006) 978-1001.

[15] C. Desceliers, C. Soize, R. Ghanem, Identification of chaos representations of elastic properties of random media using experimental vibration tests, Computational Mechanics, 39(6) (2007) 831-838.

[16] S. Finsterle, J. Najita, Robust estimation of hydrogeologic model parameters, Water Resources Research, 34(11) (1998) 2939-2947.

[17] R. Ghanem, P.D. Spanos, Stochastic Finite Elements: A spectral Approach, Spinger-verlag, New-York, 1991 (revised edition, Dover Publications, New York, 2003).

[18] R. Ghanem R., S. Dham, Stochastic finite element analysis for multiphase flow in heterogeneous porous media, Transp. Porous Media 32 (1998) 239-262.

[19] R. Ghanem, R. Doostan, Characterization of stochastic system parameters from experimental data: A bayesian inference approach, Journal of Computational Physics, 217(1) (2006) 63-81.

[20] R. Ghanem, R. Doostan, J. Red-Horse, A probability construction of model validation, Computer Methods in Applied Mechanics and Engineering, Vol: 197, No: 29-32, pp. 2585-2595, 2008.

[21] D. Ghosh, C. Farhat, Strain and stress computation in stochastic finite element methods, International Journal for Numerical Methods in Engineering, 74(8) (2008) 1219-1239. 
[22] J. Guilleminot, C. Soize, D. Kondo, C. Benetruy, Theoretical framework and experimental procedure for modelling volume fraction stochastic fluctuations in fiber reinforced composites, International Journal of Solid and Structures 45(21) (2008) 5567-5583.

[23] D.T. Hristopulos, Spartan Gibbs random field models for geostatistical applications, SIAM Journal on Scientific Computing, 24(6) (2003) 2125-2162.

[24] J. Kaipio, E. Somersalo, Statistical and Computational Inverse Problems, Springer-Verlag, New York, 2005.

[25] O.M. Knio, O.P. Le Maitre, Uncertainty propagation in CFD using polynomial chaos decomposition, Fluid Dynamics Research 38(9) (2006) 616-640.

[26] C.L. Lawson, R.J. Hanson, Solving Least Squares Problems, Prentice-Hall, 1974

[27] H.K.H. Lee, D.M. Higdon, Z.O. Bi, M.A.R. Ferreira, M. West, Markov random field models for high-dimensional parameters in simulations of fluid flow in porous media, Technometrics, 44(3) (2002) 230-241.

[28] H.K.H. Lee, D.M. Higdon, C.A. Calder, C.H.H. Holloman, Efficient models for correlated data via convolutions of intrinsic processes, Statistical Modelling, 5(1) (2005) 53-74.

[29] O.P. Le Maitre, A Newton method for the resolution of steady stochastic Navier-Stokes equations, Computers and Fluids, 38(8) (2009) 1566-1579.

[30] O.P. Le-Maitre, O.M. Knio, Spectral Methods for Uncertainty Quantification with Applications to Computational Fluid Dynamics, Springer, Heidelberg, 2010.

[31] X. Ma, N. Zabaras, An efficient Bayesian inference approach to inverse problems based on an adaptive sparse grid collocation method, Inverse Problems, 25(3) (2009) Article Number: 035013.

[32] Y.M. Marzouk, H.N. Najm, L.A. Rahn, Stochastic spectral methods for efficient Bayesian solution of inverse problems, Journal of Computational Physics 224(2) (2007) 560-586. 
[33] Y.M. Marzouk, H.N. Najm, Dimensionality reduction and polynomial chaos acceleration of Bayesian inference in inverse problems, Journal of Computational Physics, 228(6) (2009) 1862-1902.

[34] H.N. Najm, Uncertainty quantification and polynomial chaos techniques in computational fluid dynamics, Journal Review of Fluid Mechanics 41 (2009) $35-52$.

[35] A. Nouy, Generalized spectral decomposition method for solving stochastic finite element equations: Invariant subspace problem and dedicated algorithms, Computer Methods in Applied Mechanics and Engineering, 197(51-52) (2008) 4718-4736.

[36] A. Nouy, A. Clement, F. Schoefs, et al, An extended stochastic finite element method for solving stochastic partial differential equations on random domains, Computer Methods in Applied Mechanics and Engineering 197(51-52) (2008) 4663-4682.

[37] J.R. Red-Horse, A.S. Benjamin, A probabilistic approach to uncertainty quantification with limited information, Reliability Engineering and System Safety 85 (2004) 183190.

[38] G.I. Schueller (Ed.), Computational methods in stochastic mechanics and reliability analysis, Computer Methods in Applied Mechanics and Engineering 194(12-16) (2005) 1251-1795.

[39] G.I. Schueller, On the treatment of uncertainties in structural mechanics and analysis, Computers and Structures 85(5) (2007) 235-243.

[40] R .J. Serfling, Approximation Theorems of Mathematical Statistics, John Wiley \& Sons, 1980.

[41] C. Soize, R. Ghanem, Physical systems with random uncertainties : Chaos representation with arbitrary probability measure, SIAM Journal on Scientific Computing 26(2) (2004) 395-410.

[42] C. Soize, Random matrix theory for modeling uncertainties in computational mechanics, Computer Methods in Applied Mechanics and Engineering, 194(12-16) (2005) 1333-1366. 
[43] C. Soize, Non Gaussian positive-definite matrix-valued random fields for elliptic stochastic partial differential operators, Computer Methods in Applied Mechanics and Engineering, 195 (2006), 26-64.

[44] C. Soize, Tensor-valued random fields for meso-scale stochastic model of anisotropic elastic microstructure and probabilistic analysis of representative volume element size, Probabilistic Engineering Mechanics 23 (2008) 307-323.

[45] C. Soize, Construction of probability distributions in high dimension using the maximum entropy principle. Applications to stochastic processes, random fields and random matrices, International Journal for Numerical Methods in Engineering 76(10) (2008) 1583-1611.

[46] C. Soize, R. Ghanem, Reduced chaos decomposition with random coefficients of vector-valued random variables and random fields, Computer Methods in Applied Mechanics and Engineering, 198(21-26) (2009), 1926-1934.

[47] C. Soize, Generalized probabilistic approach of uncertainties in computational dynamics using random matrices and polynomial chaos decompositions, International Journal for Numerical Methods in Engineering 81(8) (2010) 939970.

[48] C. Soize, Identification of high-dimension polynomial chaos expansions with random coefficients for non-Gaussian tensor-valued random fields using partial and limited experimental data, Computer Methods in Applied Mechanics and Engineering, 199(33-36) (2010) 2150-2164.

[49] C. Soize, C. Desceliers, Computational aspects for constructing realizations of polynomial chaos in high dimension, SIAM Journal on Scientific Computing, 32(5) (2010) 2820-2831.

[50] J.C. Spall, Introduction to Stochastic Search and Optimization, John Wiley and Sons, Hoboken, New Jersey, 2003.

[51] M.T. Tan, G.-L. Tian, K.W. Ng, Bayesian Missing Data Problems, EM, Data Augmentation and Noniterative Computation, Chapman \& Hall / CRC Press, Boca Raton, 2010.

[52] G. R. Terrell, D. W. Scott, Variable kernel density estimation, The Annals of Statistics, 20(3) (1992) 1236-1265. 
[53] J.A. Vargas-Guzman, T.-C.J. Yeh, The successive linear estimator: a revisit, Advances in Water Resources, 25(7) (2002) 773-781.

[54] E. Walter, L. Pronzato, Identification of Parametric Models from Experimental Data, Springer, 1997.

[55] X.L. Wan, G.E. Karniadakis, Multi-element generalized polynomial chaos for arbitrary probability measures, SIAM Journal on Scientific Computing 28(3) (2006) 901-928.

[56] X.L. Wan, G.E. Karniadakis, Solving elliptic problems with non-Gaussian spatially-dependent random coefficients, Computer Methods in Applied Mechanics and Engineering, 198(21-26) (2009) 1985-1995

[57] J.B. Wang, N. Zabaras, A Bayesian inference approach to the inverse heat conduction problem, International Journal of Heat and Mass Transfer, 47(1718) (2004) 3927-3941.

[58] J.B. Wang, N. Zabaras, Hierarchical Bayesian models for inverse problems in heat conduction, Inverse Problems, 21(1) (2005) 183-206.

[59] N. Zabaras, B. Ganapathysubramanian, A scalable framework for the solution of stochastic inverse problems using a sparse grid collocation approach, Journal of Computational Physics, 227(9) (2008) 4697-4735. 